\title{
Revisiting the Energy-Efficient Hybrid D-A Precoding and Combining Design For mm-Wave Systems
}

\begin{tabular}{|r|l|}
\hline Journal: & IEEE Transactions on Green Communications and Networking \\
\hline Manuscript ID & TGCN-TPS-19-0047.R1 \\
\hline Manuscript Type: & Transactions Paper Submissions \\
\hline Author: & $19-$ Sep-2019 \\
\hline Complete List of Authors: & $\begin{array}{l}\text { Alluhaibi, Osama; University of Warwick, WMG } \\
\text { Kamed, Qasim } \\
\text { Kampert, Erik; University of Warwick, WMG } \\
\text { Higgins, Matthew } \\
\text { Wang, Jiangzhou }\end{array}$ \\
\hline Keyword: & \\
\hline
\end{tabular}

\section{SCHOLARONE $^{\text {m }}$ \\ Manuscripts}




\title{
Revisiting the Energy-Efficient Hybrid D-A Precoding and Combining Design For mm-Wave Systems
}

\author{
Osama Alluhaibi, Qasim Zeeshan Ahmed ${ }^{1}$, Erik Kampert, Matthew D. \\ Higgins Senior, IEEE and Jiangzhou Wang ${ }^{2}$ Fellow, IEEE, \\ WMG, University of Warwick, Coventry, UK \\ ${ }^{1}$ University of Huddersfield Queensgate, Huddersfield, UK. \\ ${ }^{2}$ School of Engineering and Digital Arts, University of Kent, Canterbury, UK.
}

\begin{abstract}
Hybrid digital to analog (D-A) precoding is widely used in millimeter wave systems to reduce the power consumption and implementation complexity incurred by the number of radio frequency (RF) chains that consume a lot of the transmitted power in this system. In this paper, an optimal number of RF chains is proposed to achieve the desired energy efficiency (EE). Here, the optimization problem is formulated in terms of fractional programming maximization, resulting in a method with a twofold novelty: First, the optimal number of RF chains is determined by the proposed bisection algorithm, which results in optimized number of data streams. Second, the optimal analog precoders/combiners are designed by eigenvalue decomposition and power iteration algorithm, followed by the digital precoders/combiners which are designed based on the singular value decomposition to the proposed effective uplink and downlink channel gains. Furthermore, the proposed D-A systems are designed carefully to attain a lower complexity than the existing D-A algorithms while achieving reasonable performance. Finally, the impact of utilizing a different number of quantized bits of resolution on the EE is investigated. Simulation results show that the proposed algorithms outperform existing algorithms in terms of EE, spectral efficiency, and computational complexity.
\end{abstract}

\section{Index Terms}

Energy efficiency, 5G, Hybrid precoding and combining, millimetre wave, massive MIMO, optimization problem.

O. Alluhaibi, E. Kampert, M. D. Higgins are with WMG, University of Warwick (email: \{o.alluhaibi, E.Kampert, M.Higgins\}@warwick.ac.uk). Q. Z. Ahmed is with School of Computing and Engineering, University of Huddersfield (email: q.ahmed@hud.ac.uk). J. Wang are with the School of Engineering and Digital Arts, University of Kent, UK (email: j.z.wang@kent.ac.uk). Part of this work has been presented in IEEE Globecom 2018. 


\section{INTRODUCTION}

Energy efficiency (EE), spectral efficiency, power consumption, and computational complexity are usually of most concern in wireless communication system design. Cooperative and relaying technologies are combined to reduce system power consumption by allowing different terminals (mobile users or dedicated relays) assist each other [1]-[3]. Cognitive radio (CR) techniques are often utilized to improve spectral efficiency that allows secondary users (SUs) to share licensed frequency bands with primary users (PUs) [4], [5]. It is expected that global mobile data traffic will reach 49 Exabytes-per-month by 2021 [6], at this rate, even with some benefits offered by $\mathrm{CR}$, exploring new frequency bands seems to be one of the effective solutions to alleviate the current scarcity of spectrum resource. Millimeter wave (mm-Wave) technology is deemed as the preferred candidate [7]-[10] to tackle the scarcity of this resource. Due to the high pathloss and shadowing effects on mm-Wave signals, conventional multiple-input multiple-output (MIMO) transceiver structures are no longer able to obtain sufficient gain for reliable signal detection. Consequently, enhancing the strength of the received signals requires an increased number of antennas at the transmitter and receiver sides. Accordingly, massive MIMO transceiver architecture is envisioned to be deployed as part of the future fifth generation $(5 \mathrm{G})$ wireless communication systems. Unfortunately, such large scale mm-Wave MIMO systems will lead to high power consumption and hardware costs, with each antenna requiring an individual RF chain and consuming $70 \%$ of the transmitted power [11]-[16]. This traditional fully digital precoding solution is prohibitively constrained in mm-Wave MIMO systems. A simpler approach is to employ a single RF chain connected to a large number of antennas as proposed in [17] for mm-Wave systems. However, this approach will lead to a solution which is less power hungry than the fully digital precoder, but its spectral efficiency performance is much lower than that of the prior system [13], [14], [18]. Accordingly, a hybrid D-A precoding system has been studied to balance the spectral efficiency and improve EE which consists of a low-dimension digital beamformer and a high-dimension analog beamformer implemented using phase shifters.

\section{A. Literature Review}

Sparse precoding based on an orthogonal matching pursuit (OMP) algorithm is proposed for the hybrid D-A mm-Wave system in [18]. OMP consumes significant power and computational complexity to achieve the required spectral efficiency maximization of the system. To achieve higher spectral efficiency than OMP, in [14] MO-AltMin algorithm for the hybrid D-A system 
was designed. They also provided the EE criteria calculations only. The number of RF chains was investigated in [19], using two new algorithms whereas the hybrid D-A system was designed based on a conventional OMP algorithm. There are two issues with this approach: firstly, it is difficult to find the optimal number of RF chains, as it is equivalent to a large number of antennas. Secondly, the hybrid D-A precoding was not optimized for the selected number of RF chains. Previously in [14], [18] and [19], neither the number of RF chains was optimized, nor was the computational complexity of the hybrid D-A precoding considered to be reduced. Taking into consideration the aforementioned issues, authors in [20] proposed an algorithm based on the Dinkelbach method (DM) with a limited feasibility to find the optimal number of RF chains. However, at the initial stage only, the DM employs the fully digital solution to solve the EE objective function which constrains the active RF chains in the system are equivalent to the number of antennas. Furthermore, the DM does not guarantee an optimal solution to the EE fractional programming maximization problem as mentioned in [21]. The complexity of the current algorithms was reduced by introducing gradient pursuit (GP) approach to design the hybrid D-A system in [20].

Minimizing the Euclidean distance between a near-optimal hybrid precoder and the fullydigital precoder can approximately maximize the spectral efficiency of the system. This renders the design of the hybrid precoder as a matrix factorization problem with a non-convex constant modulus constraint. Such optimization problems are popular and solved based on the alternating minimization technique [14], [18]-[20]. However, those non-convex constant modulus constraints for this optimization problem introduces a high complexity in solving the Euclidean distance problem. For OMP in [18], [19] when designing the analog precoder, the number of antennas was iteratively increased and the singular value decomposition (SVD) operation was also applied. The existing algorithm MO-AltMin [14] used to design the analog part comes from three stages: conjugate descent, find the local optimum, and the Kronecker product of large-sized matrices. In contrast to OMP and MO-AltMin algorithms which have high complexity, the GP algorithm in [20] avoids a matrix inversion per iteration which reduces the complexity of the system noticeably. However, GP still applies one matrix vector multiplication per iteration via SVD for designing the analog precoder which significantly increases the complexity of a system when a large number of antennas are present. For the design of the digital part in [14], [18]-[20], the pseudo inverse of a matrix dimension was applied, which equals the number of antennas in the mm-Wave system. This operation not only increases the computational complexity due 
to a large number of antennas, but also sacrifices performance when the analog beamformer is made irreversible. Consequently, the computational complexity of hybrid D-A mm-Wave systems based on the existing algorithms is always high. Hence, it is of paramount importance that the hybrid D-A mm-Wave system is designed carefully, aiming for high energy efficiency and lower computational complexity.

\section{B. Methodology and Contributions}

As noted above, in the existing hybrid D-A mm-Wave systems, neither the number of RF chains was optimized to enhance EE, nor was the reduction of the computational complexity of the hybrid D-A mm-Wave system investigated. Therefore, this paper aims to develop new algorithms that can assist in the design of energy efficient hybrid D-A mm-Wave systems, in which the number of RF chains is optimized whilst simultaneously lowering the computational complexity of the D-A precoders and combiners. Hence, the main contributions of this work are:

1) Modeling: The optimization problem is formulated as the fractional programming maximization which divides the optimization problem into two sub-problems:

a) Sub-problem One: This is formulated as a power allocation problem. Bisection algorithm (BI) is proposed for it's solution. The optimal number of RF chains is equal to the number of all the positive and non-zero power allocation values that are calculated via BI. To activate only the optimal RF chains, in the system, BI uses digital switches to activate the selected optimal RF chains. The digital switches are associated with the base band processing block, which is connected to a single RF chain. In this model, the number of data streams is the same as the number of RF chains. Subsequently, the number of data streams in the system is also optimized.

b) Sub-problem Two: This problem is focused on maximizing the spectral efficiency of the system by designing the D-A precoder and combiner matrices with low complexity. The conventional way in [14], [18]-[20] is to avoid multi-variable joint optimization and decouple the transmitter and receiver, which adds complexity to the existing algorithms. In contrast, this paper introduces the concept of effective channel gains to realize the joint design of the transceiver, and investigate the design hybrid D-A system for low energy consumption. The analog beamformer is designed under non-convex constant modulus constraints based on two methods that exploit the potential of gaining the knowledge 
of the channel. The first method is called the eigenvalue decomposition (EVD), and is based on calculating the eigenvector that corresponds to the largest eigenvalue [22]. EVD achieves high spectral efficiency but is computationally complex because of the decomposition operation. The second method is called power iteration (PI) and based on calculating the eigenvector that corresponds to the largest eigenvalue, which avoids the decomposition operation. The main advantages of the EVD and PI methods are that both schemes: i) do not require calculating the gradient of the optimization function as utilized in existing algorithms [14], [17], [18], [20], which reduces the complexity of the system significantly; ii) do not require a high number of iterations, as they only calculate the eigenvector that corresponds to the largest eigenvalue; iii) avoid the use of the Euclidean distance optimization problem. Thereafter, the digital precoder is designed by proposing a low dimension matrix that is generated based on he duality of the uplink and downlink effective channel gains. The optimal digital precoder is obtained by applying SVD to a low dimensional effective uplink and downlink channel gain matrix.

2) Complexity Reduction: The proposed methods in this paper manage to reduce the computational complexity of the hybrid D-A system. The design of the analog part based on EVD only involves the number of antennas without the need for iterative optimization and SVD operations. Then, the PI algorithm is deployed to determine the highest eigenvalue and corresponding eigenvector without the need of a decomposition step. Besides, the digital part does not require a high dimension matrix as a low dimension matrix is proposing based on the effective uplink and downlink channel gains.

3) Phase Shifters Resolution: Since the power consumption increases with the resolution of the phase shifters of the analog precoder, a trade-off between the spectral efficiency and power consumption based on a limited resolution is investigated. This allows us to conclude the $\mathrm{EE}$ of the intended hybrid D-A system when utilizing different numbers of quantized bits. The simulation results show that the EE of the proposed hybrid D-A precoding algorithms are superior to these precoders. The simulation result also allows to investigates the effects of utilizing few quantized bits on the spectral efficiency and EE and shows that the hybrid precoding with an optimal number of quantized bits of resolution attains the best EE performance. Moreover, numerical results show that the proposed hybrid D-A has a much lower complexity than the existing hybrid D-A mm-Wave system algorithms. 

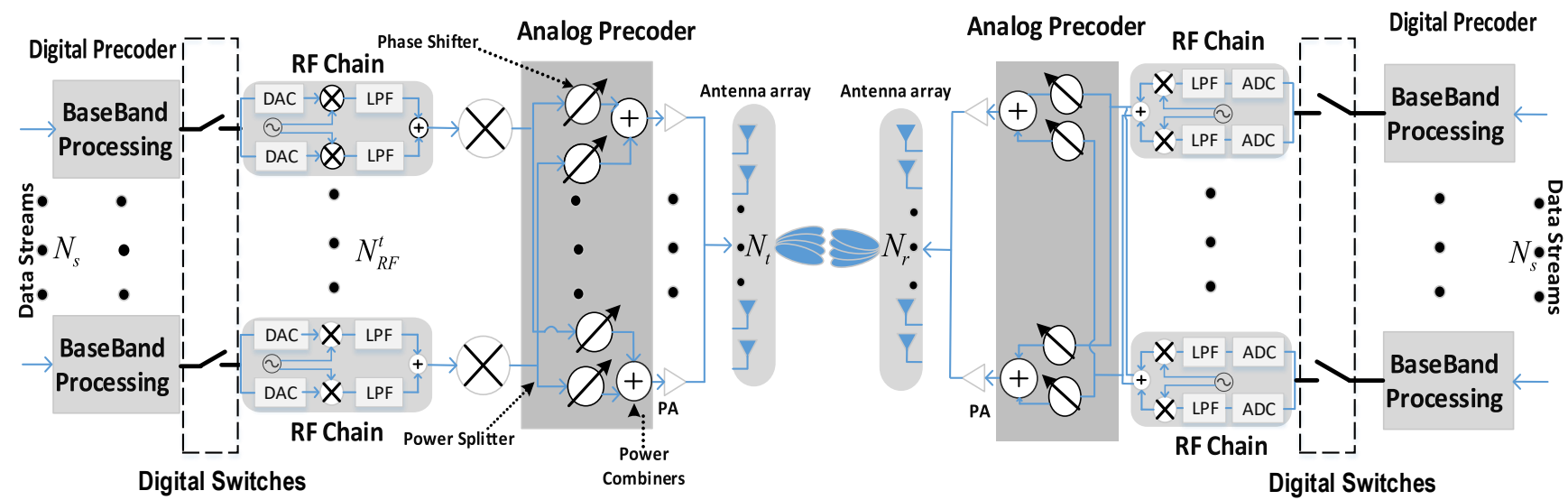

Figure 1: Block diagram of a transceiver hybrid D-A precoding and combiner mm-Wave system.

Notation: Bold uppercase letter $X$ denotes a matrix and bold lowercase letter $x$ denotes a vector. The transpose and conjugate transpose of a matrix are denoted by $(\cdot)^{T}$ and $(\cdot)^{H}$ respectively. The diagonal matrix is denoted as $\operatorname{diag}[\cdot] \cdot\|\cdot\|_{F}$ denotes Frobenius norm of a matrix, while $\|\cdot\|_{1}$ and $\|\cdot\|_{2}$ denote the 1-and 2-norm of a vector. $\arg (x)$ generates a vector containing the phases of the entries.

\section{System Model}

Fig. 1 shows a downlink massive MIMO mm-Wave system for hybrid D-A precoding. The base station (BS) sends $N_{s}$ data streams via $N_{t}$ transmit antennas to the receiver which consists of $N_{r}$ receiver antennas and has a similar implementation as the transmitter. Therefore, it is assumed that there are $N_{R F}^{t}$ and $N_{R F}^{r}$ RF chains in the BS to the receiver. Once the set of BS data streams $S$ (with size $N_{s}$ ) are precoded in the digital (baseband) side by matrix $D_{B B}$, they are connected to $N_{R F}^{t} \mathrm{RF}$ chains for the up-conversion through digital switches. Hence, the size of the matrix $D_{B B}$ should be $N_{R F}^{t} \times N_{s}$. After that, the $N_{R F}^{t}$ signals from each RF chains will be preprocessed by the analog precoder $\boldsymbol{A}_{R F}$. Finally, the mm-Wave signals are connected to $N_{t}$ antennas for transmission. It is straightforward to see that the size of matrix $A_{R F}$ is $N_{t} \times N_{R F}^{t}$. After a hybrid D-A precoding operation is applied, the signals are transmitted through the transmitting antennas and the receiver. For matrix $\boldsymbol{A}_{R F}$, this implies that the amplitude of its elements should remain constant and only the phase information is adjustable, as its corresponding precoding operation is implemented utilizing an analog phase shifter. Accordingly, the condition $\left(\boldsymbol{A}_{R F}^{(w)} \boldsymbol{A}_{R F}^{(w)}\right)_{W, W}=N_{t}^{-1}$ should hold for all $w$, which implies that all non-zero elements of $A_{R F}$ have the same amplitude, where $A_{R F}^{(w)}$ denotes the $w^{\text {th }}$ column vector of $A_{R F}$ and (.) $)_{W, W}$ denotes the $W^{t h}$ diagonal element of a matrix. Furthermore, the digital precoder should satisfy the total transmit power constraint 


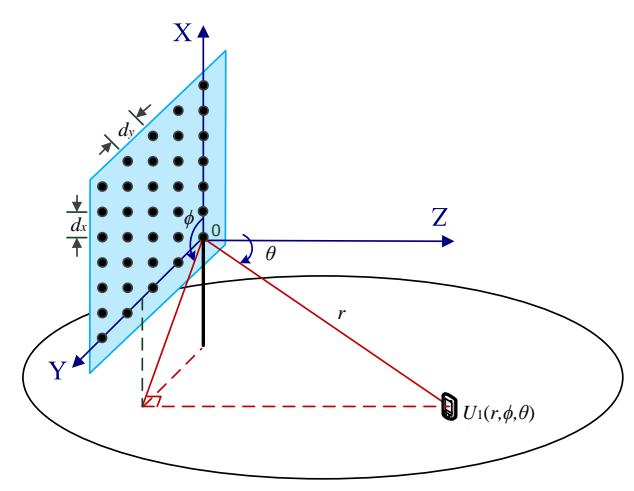

Figure 2: Introduction of spherical angles and spherical unit vectors in a Cartesian coordinate system

by normalizing $\left\|A_{R F} D_{B B}\right\|_{F}^{2}=N_{s}$. Note that, both $D_{B B}$ and $A_{R F}$ may not be square matrices as $N_{s} \leq N_{R F}^{t}$ and $N_{t}>>N_{R F}^{t}$.

\section{A. Channel Model}

The mm-Wave channel is portrayed with the popularly utilized and experimentally supported multipath channel model in which a several propagation paths are associated with a few scatterers [23], [24]. To consolidate this effect, the 3-dimensional (3D) Saleh-Valenzuela geometrystatistical channel model is adopted [23], [25], [26] which is represented as

$$
\boldsymbol{H}=\sqrt{\frac{N_{t} N_{r} P_{n_{t}}}{L}} \sum_{l=1}^{L}\left(h_{n_{r}, n_{t}, l}^{3 D} \boldsymbol{s} \boldsymbol{v}_{r}\left(\theta_{l}^{r}, \phi_{l}^{r}\right) \boldsymbol{s} \boldsymbol{v}_{t}^{H}\left(\theta_{l}^{t}, \phi_{l}^{t}\right)\right),
$$

where $H \in \mathbb{C}^{N_{r} \times N_{t}}$, and $L$ is the number of multipaths [18]. $P_{n_{t}}$ is the power of the $n_{t}$-th transmit (Tx) antenna and is calculated by assuming a single slope exponential power delay profile as in [25]. The superscript (and subscript) $t$ and $r$ represent the transmitter and receiver respectively. The $3 \mathrm{D}$ channel gain $h_{n_{r}, n_{t}}^{3 D}$ from the $n_{t}$-th Tx antenna element to the $n_{r}$-th receive antenna $(\mathrm{Rx})$ experiencing the $l$-th multipath is given by

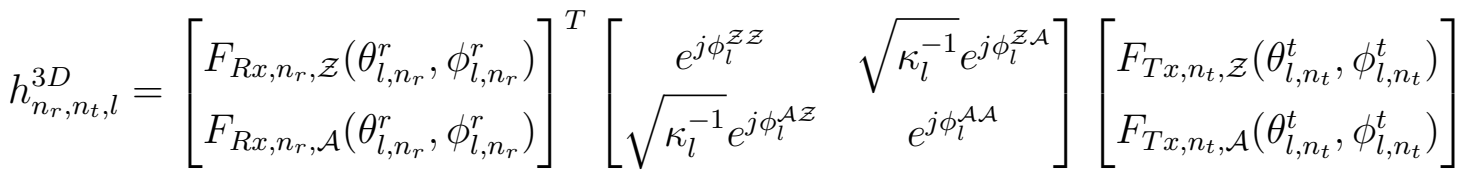

$$
\begin{aligned}
& e^{\left(j 2 \pi \lambda^{-1}\left(\overline{\boldsymbol{\varphi}}_{l, n_{r}} \overline{\boldsymbol{r}}_{R x}^{T}\right)\right)} e^{\left(j 2 \pi \lambda^{-1}\left(\overline{\boldsymbol{\phi}}_{l, n_{t}} \overline{\boldsymbol{r}}_{T x}^{T}\right)\right)},
\end{aligned}
$$

where $\lambda$ is the wavelength, and $\theta_{l}^{t}\left(\phi_{l}^{t}\right)$ and $\theta_{l}^{r}\left(\phi_{l}^{r}\right)$ represent the zenith (azimuth) angles of departure (AoD) and arrival (AoA), respectively. A local coordinate system is introduced by the 
$x, y, z$ axes, the spherical angles and the spherical unit vectors as shown in Fig. 2. $F_{R x, n_{r}, \mathcal{Z}}$ and $F_{R x, n_{r}, \mathcal{A}}$ are the Rx beam pattern for the zenith $(\mathcal{Z})$ and azimuth $(\mathcal{A})$ polarization. $F_{T x, n_{t}, \mathcal{Z}}$ and $F_{T x, n_{t}, \mathcal{A}}$, are the Tx beam pattern for the $n_{t}$-th Tx antenna. $\phi_{l}^{\mathcal{Z Z}}, \phi_{l}^{\mathcal{Z} \mathcal{A}}, \phi_{l}^{\mathcal{A Z}}$, and $\phi_{l}^{\mathcal{A A}}$ are the initial random phases for zenith $(\mathcal{Z Z})$, cross $(\mathcal{Z A}, \mathcal{A Z})$, and azimuth $(\mathcal{A A})$ polarization for the $l$-th multipath. According to $3 \mathrm{GPP}$ channel model, $\kappa_{l}$ is the cross polarization power ratio in linear scale for the lth multipath [26]. For each path, the cross polarization power ratios $\kappa_{l}$ is generated based on log-Normal distribution [26].

The receive antenna array phase offset of the $l$-th multipath is represented as $e^{\left(j 2 \pi \lambda^{-1}\left(\bar{\varphi}_{l, n_{r}} \cdot \bar{r}_{R x}^{T}\right)\right.}$ where $\bar{\varphi}_{l, n_{r}}$ is the arrival spherical unit vector and $\overline{\boldsymbol{r}}_{R x}$ is the position vector of the Rx antenna of the user located at the origin which is yielded by

$$
\begin{aligned}
& \bar{\varphi}_{l, n_{r}}=\left[\cos \vartheta_{l, n_{r}} \cos \varphi_{l, n_{r}}, \cos \vartheta_{l, n_{r}} \sin \varphi_{l, n_{r}}, \sin \vartheta_{l, n_{r}}\right], \\
& \overline{\boldsymbol{r}}_{R x}=\left[x_{R x}=N_{r_{x}} u_{x}, y_{R x}=h_{U}+N_{r_{y}} u_{y}, z_{R x}=0\right],
\end{aligned}
$$

where $\vartheta_{l, n_{r}}$ and $\varphi_{l, n_{r}}$ are the zenith and azimuth AoA. $h_{U}$ is the height of the user from the ground. $e^{\left(j 2 \pi \lambda^{-1}\left(\bar{\phi}_{l, n t} \cdot \bar{r}_{T x}^{T}\right)\right.}$ is the array phase offset for the $n_{t}$-th Tx antenna and $l$-th multipath. $\overline{\boldsymbol{\phi}}_{l, n_{t}}$ is the departure spherical unit vector of the $l$-th multipath and $n_{t}$-th Tx antenna. $\overline{\boldsymbol{r}}_{T x}$ is the position vector of the Tx antenna element relative to the user. $\overline{\boldsymbol{\phi}}_{l, n_{t}}$ and $\overline{\boldsymbol{r}}_{T x}$ are introduced as

$$
\begin{aligned}
\overline{\boldsymbol{\phi}}_{l, n_{t}} & =\left[\cos \theta_{l, n_{t}} \cos \phi_{l, n_{t}}, \cos \theta_{l, n_{t}} \sin \phi_{l, n_{t}}, \sin \theta_{l, n_{t}}\right], \\
\overline{\boldsymbol{r}}_{T x} & =\left[x_{T x}=N_{t_{x}} u_{x}, y_{T x}=h_{B}+N_{t_{y}} u_{y}, z_{T x}=0\right],
\end{aligned}
$$

where $\theta_{l, n_{t}}$ and $\phi_{l, n_{t}}$ are the zenith and azimuth AoD, and $h_{B}$ denotes the height of the BS. As the uniform planar antenna (UPA) structure [25] is a preferred choice for a $3 \mathrm{D}$ channel, $N_{t}=N_{t_{x}} \times N_{t_{y}}$, and $N_{r}=N_{r_{x}} \times N_{r_{y}}$, where $\left(N_{t_{x}}, N_{r_{x}}\right)$ and $\left(N_{t_{y}}, N_{r_{y}}\right)$ represent the numbers of antennas in the $x$-axis and $y$-axis. The array response in (1) is defined as $s v_{b}$ where $b \in\{r, t\}$ represents the arrival at the receiver $(r)$ or the departure at the transmitter $(t)$, and given by [18], [25]

$$
\boldsymbol{s} \boldsymbol{v}_{b}\left(\theta_{l}^{b}, \phi_{l}^{b}\right)=\operatorname{vec}\left[\boldsymbol{s} \boldsymbol{v}_{b_{x}}(\Psi) \boldsymbol{s} \boldsymbol{v}_{b_{y}}^{H}(\Phi)\right], \quad b \in[r, t],
$$

where the directions of the array responses $\boldsymbol{s} \boldsymbol{v}_{b_{x}}$ and $\boldsymbol{s} \boldsymbol{v}_{b_{y}}$ are represented by $\boldsymbol{s} \boldsymbol{v}_{b_{q}}$ and devised as

$$
\boldsymbol{s}_{b_{q}}(\Omega)=\frac{1}{\sqrt{N_{b_{q}}}}\left[1, e^{j \Omega}, \cdots, e^{j\left(N_{b_{q}}-1\right) \Omega}\right]^{T}, q \in[x, y]
$$


where $\Omega \in\{\Psi, \Phi\}$ and the values of $\Psi$ and $\Phi$ are given by

$$
\begin{array}{ll}
\Psi=-2 \pi \lambda^{-1} u_{x} \sin \left(\theta_{l}^{b}\right) \cos \left(\phi_{l}^{b}\right), \quad b \in[r, t], \\
\Phi=-2 \pi \lambda^{-1} u_{y} \sin \left(\theta_{l}^{b}\right) \sin \left(\phi_{l}^{b}\right), \quad b \in[r, t],
\end{array}
$$

$u_{x}$ and $u_{y}$ are the inter-element distances in the $x$ - and $y$-axes, respectively.

\section{B. Signal Model}

The received signal for all $N_{R F}$ receive antennas $\boldsymbol{y}=\left[y_{1}, y_{2}, \cdots, y_{N_{R F}}\right]^{T}$ at the user is expressed as

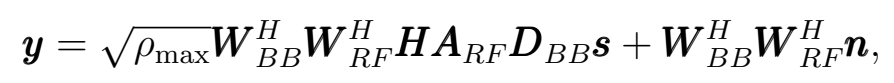

where $\rho_{\text {max }}$ represents the average received power. The symbol vector $s \in \mathbb{C}^{N_{s}}$ with $\mathbb{E}\left[s s^{H}\right]=$ $\frac{1}{N_{s}} \boldsymbol{I}_{N_{s}} . \boldsymbol{n} \in \mathbb{C}^{N_{r}}$ is the zero-mean complex Gaussian noise vector with independent and identically distributed (i.i.d) elements with $\mathbb{E}\left[\boldsymbol{n n}{ }^{H}\right]=\sigma^{2} \boldsymbol{I}_{N_{r}}$. The analog combiner is denoted as $W_{R F}$ and the digital combiner is represented as $W_{B B}$. The dimensions of $W_{R F}$ and $W_{B B}$ are $N_{r} \times N_{R F}^{r}$ and $N_{R F}^{r} \times N_{s}$. The analog combiner is realized by phase shifters that can only adjust the phase of the signal without changing its amplitude, therefore, all the non-zero elements of $W_{R F}$ have the same amplitude. The objective is to design $A_{R F}, D_{B B}, W_{R F}$ and $W_{B B}$ to maximize the spectral efficiency $C$ with respect to the optimal number of RF chains that is given by

$$
\begin{aligned}
& \underset{\boldsymbol{A}_{R F}, \boldsymbol{D}_{B B}, \boldsymbol{W}_{R F}, \boldsymbol{W}_{B B}}{\arg \max } C=\log _{2}\left|\boldsymbol{I}_{N_{s}}+\frac{P_{\max }}{N_{s} \sigma^{2}} \boldsymbol{R}^{-1} \boldsymbol{W}_{B B}^{H} \boldsymbol{W}_{R F}^{H} \boldsymbol{H} \boldsymbol{A}_{R F} \boldsymbol{D}_{B B} \boldsymbol{D}_{B B}^{H} \boldsymbol{A}_{R F}^{H} \boldsymbol{H}^{H} \boldsymbol{W}_{R F} \boldsymbol{W}_{B B}\right|,(12 \\
& \text { subject to } \\
& \left|\left(\boldsymbol{A}_{R F}\right)_{i, j}\right|=1, \quad\left|\left(\boldsymbol{W}_{R F}\right)_{i, j}\right|=1, \quad \forall i, j, \\
& \left\|\boldsymbol{A}_{R F} \boldsymbol{D}_{B B}\right\|_{F}^{2}=N_{s},\left\|\boldsymbol{W}_{R F} \boldsymbol{W}_{B B}\right\|_{F}^{2}=N_{s},
\end{aligned}
$$

where $P_{\max }$ represents the maximum allocated power and $R^{-1}=W_{B B}^{H} W_{R F}^{H} W_{R F} W_{B B}$ is the processed noise covariance matrix. Constraint (12a) directly leads to the maximum spectral efficiency of the hybrid D-A precoding system. Therefore, the spectral efficiency of the hybrid D-A fully-connected antenna array is compared with the fully digital system [14], [18]. Constraint (12b) is the constant modulus constraint, restricting all the non-zero elements to the same amplitude, whereas (12c) represents the normalized transmit power constraint. The main challenge in solving the above problem is that it is a non-convex optimization problem since (12b) is non-convex. Furthermore, the scenario is considered in which the BS transmits signals 
to a single user. Before transmission, the parallel data streams $S$ should be preprocessed by a digital precoder and an analog precoder, respectively. Correspondingly, the received signals should also be processed by an analog and a digital combiner respectively whereafter signal detection and other operations are carried out. As the operation of the receiver is similar to that of the base station, only the precoding principle of the transmitter is presented here, and the combining process at the receiver is omitted for brevity.

\section{The Proposed Structure Design of EE Hybrid D-A MM-WaVe Systems}

As the spectral efficiency is a crucial function for designing the EE of the hybrid D-A mmWave system, maximizing $C$ is one of the main targetz of this paper. Taking into consideration the difficulties of directly designing the precoders, combiners, and the optimal number of RF chains, the problems can be partitioned into three sub-problems:

1) Designing the precoders $\boldsymbol{A}_{R F}$ and $\boldsymbol{D}_{B B}$.

2) Designing the combiners $W_{R F}$ and $W_{B B}$.

3) Designing the optimal number of RF chains $N_{R F}^{t}=N_{R F}^{r}$.

It can be seen that the first two steps demand designing the precoder matrices $A_{R F}$ and $D_{B B}$ and the combiner matrices $W_{R F}$ and $W_{B B}$ of the hybrid D-A mm-Wave system. Then the optimal number of RF chains is computed to the EE hybrid D-A mm-Wave system.

To attain the previous scenario, a platform structure with three Phases is proposed here; Phase-I At the initial stage, the spectral efficiency $C$ is maximized by designing the precoder and combiner matrices based on the assumption that all the available RF chains $N_{R F}^{t}$ are activated in the hybrid D-A mm-Wave system; Phase-II Searching for an optimal number of RF chains $N_{R F}^{t}=N_{R F}^{r}$ in the system by solving the EE fractional programming maximization problem; Phase-III Re-design the precoders and combiners matrices based on the optimal number of RF chains $N_{R F}^{t}=N_{R F}^{r}$ in the hybrid D-A mm-Wave system.

In the following sections designing the precoder, combiner matrices and the optimal number of RF chains algorithms are proposed for designing the EE hybrid D-A mm-Wave system.

\section{Precoders and Combiners Design for Hybrid D-A mM-Wave Large Antenna ARRAY SYSTEM}

In this section, the main focus is to design the hybrid D-A precoding and combining methods. Initially, the analog precoder and combiner $A_{R F}$ and $W_{R F}$ are designed based on EVD and PI 
algorithms and followed by the digital precoder and combiner $D_{B B}$ and $W_{B B}$ that exploit the duality of the effective uplink and downlink channel gains and satisfy the non-convex constraint and the total transmit power constraints. The advantages of the proposed algorithms are:

1) There is no requirement for a gradient of an objective function to determine the optimal analog and digital precoder and combiner. This helps the system to reduce the complexity in finding the optimal solution for this optimization problem as compared to the existing algorithms in [14], [17], [18], especially when large number of antennas are involved.

2) Normal optimal spectral efficiency is guaranteed by avoiding the use of Euclidean distance between the optimal fully digital system and the hybrid D-A system as required in the conventional algorithms [14], [18].

Let us first look into the design of analog precoders and combiners followed by desiging digital precoders and combiners.

\section{A. Analog Precoder and Combiner Algorithms}

Two algorithms to design the analog precoder and combiner matrices are proposed in this section. Initially, the EVD method is proposed which achieves high performance as shown in Section-VII. However, a large number of antennas in the mm-Wave system increases the computational complexity of the EVD method, which might affect its practical implementation. Therefore, the modified PI algorithm is proposed which employs lower computational complexity and achieves near-optimal performance. The design of the analog precoder $\boldsymbol{A}_{R F}$ and combiner $W_{R F}$ matrices are proposed as follows.

1) EVD scheme: Suppose $\mathbf{P}_{T}=\boldsymbol{H}^{H} \boldsymbol{H}$ where $\mathbf{P}_{T} \in \mathbb{C}^{N_{t} \times N_{t}}$, is a Hermitian diagonalizable matrix. Here, only need EVD is used to decompose $\mathbf{P}_{T}$ and then the eigenvector $\mathbf{u}_{N_{t}}$ is selected that corresponds to the largest eigenvalue. Therefore, $\mathbf{P}_{T}=\boldsymbol{U}_{T} \boldsymbol{\Lambda}_{T} \boldsymbol{U}_{T}^{H}$, where $\boldsymbol{\Lambda}_{T}$ denotes a diagonal matrix with diagonal elements that represent the eigenvalues $\gamma_{N_{t}}$, and each column of $\boldsymbol{U}_{T}$ denotes an eigenvector of $\mathbf{P}_{T}$. In this case $\mathbf{P}_{T}$ will consist of $N_{t}$ linear independent eigenvectors $\mathbf{u}_{1}, \mathbf{u}_{2}, \cdots, \mathbf{u}_{N_{t}}$ where $\mathbf{u}_{N_{t}} \in \mathbb{C}^{N_{t} \times 1}$ with corresponding eigenvalues of $\gamma_{1}, \gamma_{2}, \cdots, \gamma_{N_{t}}$, such that $\mathbf{P}_{T} \mathbf{u}_{e}=\gamma_{e} \mathbf{u}_{e}$ for $e=1, \cdots, N_{t}$.

After finding the eigenvectors that are corresponding to the largest eigenvalue, the analog precoder $\boldsymbol{A}_{R F}$ matrix can be designed with $N_{R F}^{t}$ column vectors such that $\boldsymbol{A}_{R F}=\left[\boldsymbol{a}_{1}, \boldsymbol{a}_{2}, \cdots, \boldsymbol{a}_{N_{R F}^{t}}\right]$ where $\boldsymbol{a}_{i} \in \mathbb{C}^{N_{t} \times 1}$ and $i$ is the index of the RF-th chain where $i=1,2, \cdots, N_{R F}^{t}$. In this case, the first column of the analog precoder $\boldsymbol{a}_{1}$ is designed by the eigenvector corresponding to the largest 
eigenvalue that is selected from the output of EVD method. The element-wise normalization is satisfied for the non-convex constraint in $(12 b)$ as

$$
\boldsymbol{a}_{1}=\frac{1}{\sqrt{N_{t}}} e^{j \arg \left[\boldsymbol{u}_{1}\right]}
$$

After obtaining $\boldsymbol{a}_{1}$, to design the next analog precoding vector $\boldsymbol{a}_{2}$, the first column vector of the matrix $\mathbf{P}=H^{H} \boldsymbol{H}$ is replaced by the selected eigenvector that corresponds to the largest eigenvalue from previous EVD of $\boldsymbol{a}_{1}$, therefore, EVD method are repeated. Finally, the second analog vector $\boldsymbol{a}_{2}$ is designed by using equation (13). Similarly, all the columns of the optimal analog precoder matrix $\boldsymbol{A}_{R F}^{\text {opt }}$ are designed.

For designing the analog combiner matrix $W_{R F}=\left[\boldsymbol{w}_{1}, \boldsymbol{w}_{2}, \cdots, \boldsymbol{w}_{N_{R F}^{r}}\right]$, the same steps are followed which as for the design of the analog precoder $\boldsymbol{A}_{R F}$ based on EVD. Therefore, suppose $\mathbf{P}_{R}=\boldsymbol{H}_{R}^{H} \boldsymbol{H}_{R}$ where $\mathbf{P}_{R} \in \mathbb{C}^{N_{r} \times N_{r}}$ as $\boldsymbol{H}_{R} \in \mathbb{C}^{N_{t} \times N_{r}}$, is a Hermitian and the diagonalizable matrix. Then, decomposing $\mathbf{P}_{R}$ by using EVD, then, the eigenvector $\mathbf{u}_{N_{r}}$ that corresponds to the largest eigenvalue is selected. Thus, the analog combiner is obtained as

$$
\boldsymbol{w}_{1}=\frac{1}{\sqrt{N_{r}}} e^{j \arg \left[\boldsymbol{u}_{1}\right]} .
$$

In order to design the next analog combiner vector $w_{2}$, the first column vector of the matrix $\mathbf{P}_{R}=\boldsymbol{H}_{R}^{H} \boldsymbol{H}_{R}$ is replaced by the selected eigenvector that corresponds to the largest eigenvalue from the previous EVD of $\boldsymbol{w}_{1}$. Then, the second analog vector $\boldsymbol{w}_{2}$ is designed by using equation (14). Similarly, all the columns of the optimal analog combiner matrix $W_{R F}^{o p t}$ are designed.

2) PI algorithm: The PI algorithm 1 is an iterative algorithm. At iteration $\alpha$, it multiplies the diagonalized matrix $\mathbf{P}=\boldsymbol{H}^{H} \boldsymbol{H}$ where $\mathbf{P} \in \mathbb{C}^{N_{t} \times N_{t}}$ with an iteratively normalized vector $\mathbf{v}^{\alpha-1} \in$ $\mathbb{C}^{N_{t} \times 1}$ obtained in the previous iteration $(\alpha-1)$. $\mathbf{P}$ consist of $N_{t}$ linear independent eigenvectors $\mathbf{u}_{1}, \mathbf{u}_{2}, \cdots, \mathbf{u}_{N_{t}}$ where $\mathbf{u}_{N_{t}} \in \mathbb{C}^{N_{t} \times 1}$ with corresponding eigenvalues of $\gamma_{1}, \gamma_{2}, \cdots, \gamma_{N_{t}}$, such that $\mathbf{P} \mathbf{u}_{e}=\gamma_{e} \mathbf{u}_{e}$ for $e=1, \cdots, N_{t}$. For the convergence analysis, those eigenvalues are organized such that $\left|\gamma_{1}\right| \geq\left|\gamma_{2}\right| \geq \cdots \geq\left|\gamma_{N_{t}}\right|$, where $\gamma_{1}$ is the dominant eigenvalue with a corresponding eigenvector of $\mathbf{u}_{1}$. At the start of the PI algorithm, the vector $\mathbf{v}^{0}$ is initialized randomly, and normalised such that $\left\|\mathbf{v}^{0}\right\|=1$ and $\mathbf{v}^{0^{H}} \mathbf{u}_{1} \neq 0$. Then a sequence of vectors is $\mathbf{v}^{1}, \mathbf{v}^{2}, \cdots$ is produced by continuously multipling by $\mathbf{P}$, yielding

$$
\mathbf{v}^{\alpha}=\mathbf{P} \mathbf{v}^{(\alpha-1)}=\mathbf{P}^{\alpha} \mathbf{v}^{0}
$$


where $\alpha=1,2, \cdots$ is denoted as the number of iterations. Because $\mathbf{P}$ is diagonalizable matrix, thus each vector is a linear combination of the eigenvectors and represented as

$$
\mathbf{v}^{0}=c_{1} \mathbf{u}_{1}+c_{2} \mathbf{u}_{2}+\cdots+c_{N_{t}} \mathbf{u}_{N_{t}}
$$

where $c_{1} \neq 0$ is a normalizing constant to avoid $\mathbf{u}_{e}$ from getting too large or too small. Then,

$$
\begin{aligned}
\mathbf{v}^{\alpha} & =\mathbf{P}^{\alpha} \mathbf{v}^{0} \\
& =c_{1} \gamma_{1}^{\alpha} \mathbf{u}_{1}+c_{2} \gamma_{2}^{\alpha} \mathbf{u}_{2}+\cdots+c_{N_{t}} \gamma_{N_{t}}^{\alpha} \mathbf{u}_{N_{t}}, \\
& =\gamma_{1}\left[c_{1} \mathbf{u}_{1}+\left(\frac{\gamma_{2}}{\gamma_{1}}\right) c_{2} \mathbf{u}_{2}+\cdots+\left(\frac{\gamma_{N_{t}}}{\gamma_{1}}\right) c_{N_{t}} \mathbf{u}_{N_{t}}\right] \\
& =\gamma_{1}^{\alpha}\left[c_{1} \mathbf{u}_{1}+\left(\frac{\gamma_{2}}{\gamma_{1}}\right)^{\alpha} c_{2} \mathbf{u}_{2}+\cdots+\left(\frac{\gamma_{N_{t}}}{\gamma_{1}}\right)^{\alpha} c_{N_{t}} \mathbf{u}_{N_{t}}\right]
\end{aligned}
$$

As $\gamma_{e} / \gamma_{1}<1$ for $e=2, \cdots, N_{t}$, it can be inferred that the coefficients of $\mathbf{u}_{e}$ with $e=2, \cdots, N_{t}$, converge to zero as $\alpha \rightarrow \infty$. Therefore, as $\alpha$ increases the vector $\mathbf{P}^{\alpha} \mathbf{v}^{0}$ tends to converge to the eigenvector $\mathbf{u}_{1}$. To improve the accuracy of an approximate eigenpair $\mathbf{v}^{\alpha}, \gamma^{\alpha}$, the residual vector is calculated as

$$
\mathbf{r}=\mathbf{P} \mathbf{v}^{\alpha}-\gamma_{1}^{\alpha} \mathbf{v}^{\alpha}
$$

This information assists in selecting stopping criterion for PI when

$$
\left|\gamma^{\alpha}-\gamma^{\alpha-1}\right| \leqslant \|\left.\mathbf{r}\right|_{2}
$$

is met. The main drawback of the PI algorithm is that if the initial random vector $\mathbf{v}^{0}$ is orthogonal to the eigenvector $\mathbf{u}_{1}$ then this algorithm diverges from the exact solution. In order to in this paper to avoid this drawback, initially the argument between the initializing vector $\mathbf{v}^{0}$ and the vector $\mathbf{u}_{1}$ is initially found from matrix $\mathbf{P}$, and calculated as

$$
\cos \angle\left(\mathbf{v}^{0}, \mathbf{u}_{1}\right)=\frac{\mathbf{v}^{0^{H}} \mathbf{u}_{1}}{\left\|\mathbf{v}^{0}\right\|_{2}\left\|\mathbf{u}_{1}\right\|_{2}} .
$$

When $\mathbf{v}^{0}$ and $\mathbf{u}_{1}$ are perpendicular to each other, $\cos \angle\left(\mathbf{v}^{0}, \mathbf{u}_{1}\right)=0$, and a new $\mathbf{v}^{0}$ is initialised. On the other hand, when $\cos \angle\left(\mathbf{v}^{0}, \mathbf{u}_{1}\right) \neq 0$, the algorithm is triggered to determine the largest eigenvalue with its corresponding eigenvector. The convergence rate of the PI algorithm depends on the ratio between the eigenvalues $\left|\frac{\gamma_{2}}{\gamma_{1}}\right|$. This may leads the PI to converge slowly when $\gamma_{2}$ is almost as large as $\gamma_{1}$ [27]. In the proposed PI this problem is solved by normalizing the vector $\mathbf{v}^{\alpha}$ at each iteration to avoid PI having slower convergence. The normalization step is defined as

$$
\mathbf{v}^{\alpha+1}=\frac{\mathbf{P v}^{\alpha}}{\left\|\mathbf{P} \mathbf{v}^{\alpha}\right\|_{2}}
$$




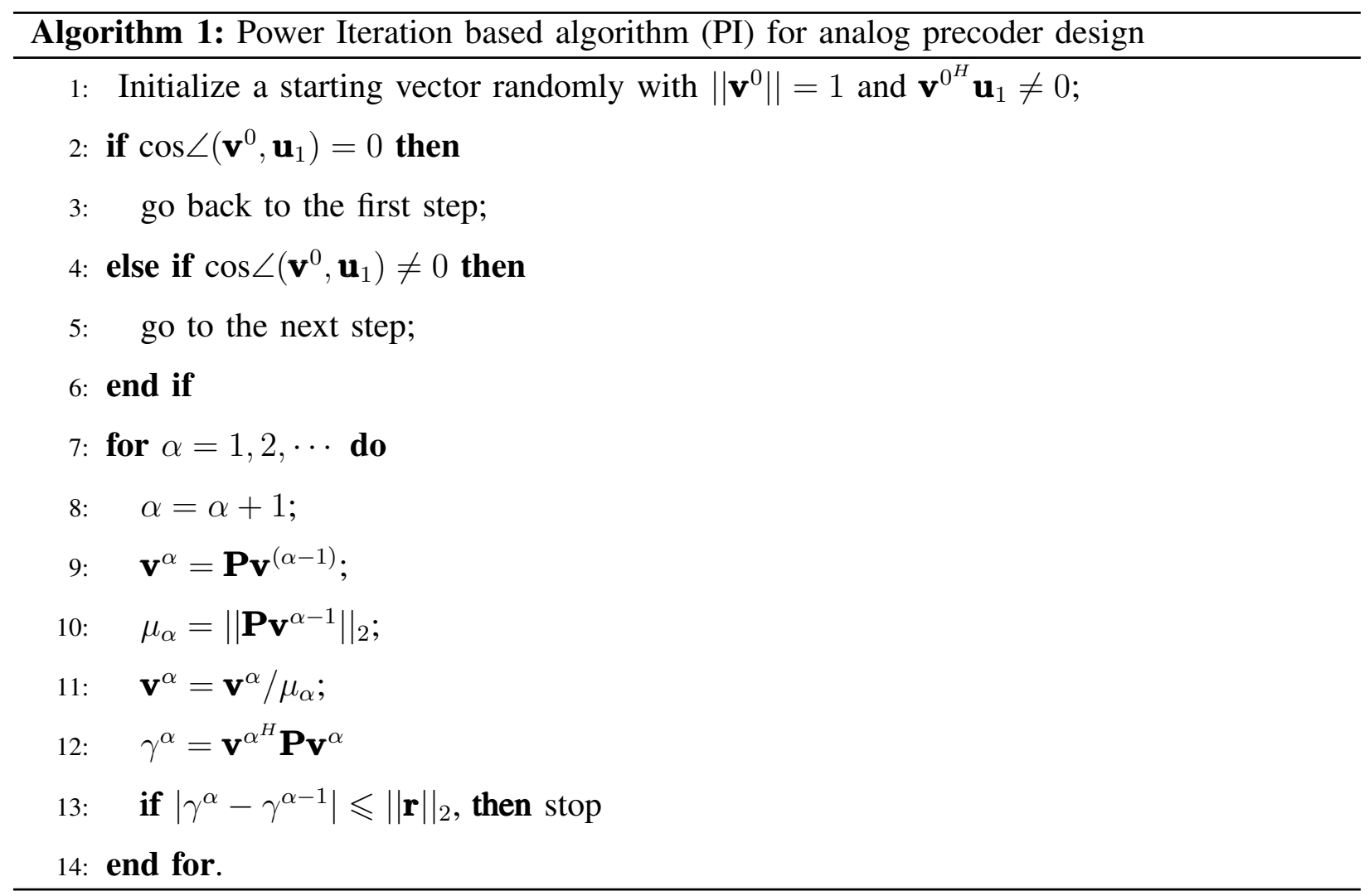

The algorithm 1 is summarized at the top of this page. Now, the same method that is used in EVD algorithm can be utilized here. Thus the analog precoder $\boldsymbol{A}_{R F}$ matrix can be designed with $N_{R F}^{t}$ column vectors such that $\boldsymbol{A}_{R F}=\left[\boldsymbol{a}_{1}, \boldsymbol{a}_{2}, \cdots, \boldsymbol{a}_{N_{R F}^{t}}\right]$ where $\boldsymbol{a}_{i} \in \mathbb{C}^{N_{t} \times 1}$ and $i$ is the index of the RF-th chain. In this case, the first column of the analog precoder $a_{1}$ is designed by the eigenvector corresponding to the largest eigenvalue with satisfaction of constant-modulus constraint in $(12 b)$ by

$$
\boldsymbol{a}_{1}=\frac{1}{\sqrt{N_{t}}} e^{j \arg \left[\boldsymbol{u}_{1}\right]}
$$

After obtaining $\boldsymbol{a}_{1}$, designing the next analog vector $\boldsymbol{a}_{2}$ is operated by replacing the the first column of $\mathbf{P}$ with the selected eigenvector that corresponds to the largest eigenvalue from previous PI of $\boldsymbol{a}_{1}$. Step 7 till step 14 as mentioned in algorithm 2 are repeated. Finally, the second analog vector $a_{2}$ is designed by using equation (22). Hence, all the columns of the optimal analog matrix $A_{R F}^{\text {opt }}$ are designed. 


\section{B. Quantized Analog Precoder and Combiner Matrices}

In the previous subsection, the analog precoder and combiner are designed assuming that the resolution of the phase shifters (PSs) elements to be infinity $\infty$. However, this assumption affects the power consumption proportionally, because the number of PSs scales linearly with the number of antennas in the system [13], [14]. Utilizing low-resolution of PSs can enhance the EE of the hybrid D-A mm-Wave system. Therefore, the phase $\vartheta$ of each elements of the analog precoder $\boldsymbol{A}_{R F}$ and combiner $\boldsymbol{W}_{R F}$ is quantized up to $B$ bits of precision. The minimum Euclidean distance is formulated between the phase of the nearest neighbour element to its quantized value in order to accurately design the quantized phases of precision. The quantized phase of each element of the analog precoder $A_{R F}$ and analog combiner $W_{R F}$ is given as

$$
\hat{\vartheta}=\frac{2 \pi \hat{\tau}}{2^{B}}, \quad \hat{\tau}=\underset{\tau \in\left[0,2 \pi\left(\frac{1}{2^{B}}\right), \cdots, 2 \pi\left(\frac{2^{B}-1}{2^{B}}\right)\right]}{\arg \min }|\vartheta-\tau|,
$$

where $\tau$ is the set of quantized phases. Finally, the analog precoder and combiner $A_{R F}^{F^{o p t}}(\hat{\vartheta})$ and $W_{R F}^{F^{o p t}}(\hat{\vartheta})$ are designed with quantized phases of each element.

\section{Digital Precoder and Combiner Methods}

After having accomplished the design of the analog beamformer, the design of the digital precoder and combiner is now in focus. The design the digital precoder and combiner with low complexity is based on the duality of the effective uplink and downlink channel gain. In general, the existing algorithms [14], [18], [20] use the well-known least square (LS) solution, which is given by [14], [18], [20]

$$
\boldsymbol{D}_{B B}=\left(\boldsymbol{A}_{R F}^{F^{o p t}{ }^{H}}(\hat{\vartheta}) \boldsymbol{A}_{R F}^{F^{o p t H}}(\hat{\vartheta})\right)^{-1} \boldsymbol{A}_{R F}^{F^{o p t} H}(\hat{\vartheta}) \boldsymbol{F}_{o p t},
$$

where $\boldsymbol{F}_{\text {opt }}$ is the optimal fully digital precoder obtained through the SVD operation of the channel matrix. The solution in (24) employs pseudo -inversion, which not only increases the computational complexity, but also sacrifices performance when the analog beamformer is irreversible. In this case, to propose low complexity and asymptotically optimal digital precoders and combiners, the optimal $D_{B B}^{o p t}$ and $\boldsymbol{W}_{B B}^{o p t}$ are designed based on the duality of the effective uplink and downlink channel gains that are denoted as $\boldsymbol{H}^{E F}$, where $\boldsymbol{H}^{E F}=\boldsymbol{W}_{R F}^{F^{o p t} H}(\hat{\vartheta}) \boldsymbol{H} \boldsymbol{A}_{R F}^{F^{o p t}}(\hat{\vartheta}), \boldsymbol{H}^{E F} \in$

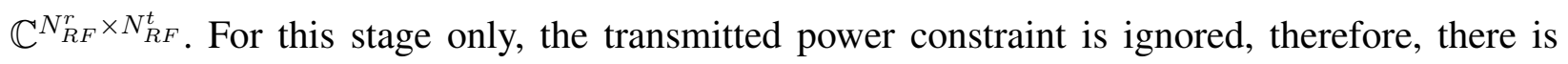


no restriction on $D_{B B}$ and $W_{B B}$. Based on this assumption, the proposed unconstrained digital precoder and combiner $D_{B B}$ and $W_{B B}$ are obtained through

$$
\begin{gathered}
\boldsymbol{D}_{B B}=\boldsymbol{V}^{E F}\left(1: N_{s}\right)=\left[\boldsymbol{v}_{1}^{E F}, \cdots, \boldsymbol{v}_{N_{s}}^{E F}\right], \\
\boldsymbol{W}_{B B}=\boldsymbol{U}^{E F}\left(1: N_{s}\right)=\left[\boldsymbol{u}_{1}^{E F}, \cdots, \boldsymbol{u}_{N_{s}}^{E F}\right],
\end{gathered}
$$

where $V^{E F}$ and $U^{E F}$ are the unitary matrices that are derived by taking SVD of the effective channel gains $H^{E F}=U^{E F} \sum^{E F} V^{E F^{H}}$. After obtaining the digital precoder and combiner from (25), and (26), both should be normalized by using a normalization step which is formulated as $D_{B B}^{o p t}=\frac{\sqrt{N_{s}} \boldsymbol{D}_{B B}}{\left\|\boldsymbol{A}_{R F}^{F^{\text {opt }} H}(\hat{\vartheta}) \boldsymbol{D}_{B B}\right\|_{F}}$, and $\boldsymbol{W}_{B B}^{\text {opt }}=\frac{\sqrt{N_{s}} \boldsymbol{W}_{B B}}{\left\|\boldsymbol{W}_{R F}^{F^{o p t}{ }^{H}}(\hat{\vartheta}) \boldsymbol{W}_{B B}\right\|_{F}}$. This normalization step guarantees that the transmitted power constraint $\left\|\boldsymbol{A}_{R F} \boldsymbol{D}_{B B}\right\|_{F}^{2}=N_{s}$ and $\left\|\boldsymbol{W}_{R F} \boldsymbol{W}_{B B}\right\|_{F}^{2}=N_{s}$ in (12c) is satisfied in the system.

\section{SeArching For An Optimal Number of RF Chains For Hybrid D-A Precoding}

\section{AND COMBINING MM-WAVE SYSTEMS}

In this section, an approach which aims at the maximization of the EE by applying the dynamic power allocation in the digital domain is proposed. Generally, in mm-Wave systems, the spectral efficiency is improved by increasing the number of RF chains which consumes up to $70 \%$ of the total transceiver power [13], [20], [28]. Therefore, an EE criterion is required which is given as [22]

$$
\gamma_{E E}=\frac{C}{P_{\text {total }}},
$$

where $P_{\text {total }}$ is the total power consumed in Watts by the transmitter and receiver hybrid D-A mm-Wave system. Hence, the total power consumed by the transmitter and receiver hybrid D-A is representing by $P_{\text {Ttotal }}$ and $P_{\text {Rtotal }}$ respectively, yielding

$$
\begin{aligned}
P_{\text {Ttotal }} & =\beta \operatorname{tr}\left(\boldsymbol{G} \boldsymbol{G}^{H}\right)+N_{R F}^{t}\left(P_{D A C}+P_{R F}\right)+2 P_{\Phi} \\
& +N_{t}\left(P_{L N A}+P_{S P}+P_{P A}+N_{R F}^{t} P_{P S}\right)+P_{B P}, \\
P_{\text {Rtotal }} & =\beta \operatorname{tr}\left(\boldsymbol{W} \boldsymbol{W}^{H}\right)+N_{R F}^{r}\left(P_{A D C}+P_{R F}\right)+2 P_{\Phi} \\
& +N_{r}\left(P_{L N A}+P_{S P}+P_{P A}+N_{R F}^{r} P_{P S}\right)+P_{B P},
\end{aligned}
$$

where $\boldsymbol{G}=\boldsymbol{A}_{R F} \boldsymbol{D}_{B B}$ and $\boldsymbol{W}=\boldsymbol{W}_{R F} \boldsymbol{W}_{B B}, \beta$ denotes the reciprocal of the amplifier efficiency. The power amplifier is modelled as $P_{P A}=P_{n_{t}} / \gamma_{P A} . P_{D A C}$ and $P_{A D C}$ in (28) and (29) are 
the power of the digital-to-analog converter and analog-to-digital converter, respectively. In (28) and (29) the following parameters $P_{L N A}, P_{S P}$ and $P_{\Phi}$ are representing the low noise amplifier per antenna, the spliter consumed power per antenna, and the common power, respectively [28]. $P_{B P}$ is the base-band processing power, and $P_{P S}$ is the power consumed by the phase shifters (including the energy for the excitation and for the compensation of insertion loss [29]). $\gamma_{P A}$ is the average transmitting power of each antenna, and is fixed to 0.375 [13], [30]. In (28) and (29), $P_{R F}$ is the power consumption of each RF chain and is modelled as

$$
P_{R F}=P_{\text {mixer }}+P_{L O}+P_{\text {filter }}+P_{B A}
$$

where $P_{\text {mixer }}$ is the power consumed by the mixer and $P_{L O}$ is the power required by the local oscillator. From (30), it can be observed that the mm-Wave system also considers the power consumed by the filter $P_{\text {filter }}$ and the base-band amplifier $P_{B A}$ which was not included in [13], [14] and [30] making the power consumption model more accurate and generalized.

\section{A. Problem Formulation}

The objective is to maximize (27) when different constraints are applied on the hybrid D-A mm-Wave system. Notice here that, the main focus of this work is on the transmitter side, which can be easily applied to the receiver side and helps in simplifying the optimization problem. In this case, for the transmitted side the spectral efficiency $C$ in (12a) can be reformulated as

$$
C=\log _{2}\left|\boldsymbol{I}_{N_{s}}+\frac{1}{\sigma^{2}} \boldsymbol{H} \boldsymbol{G} \boldsymbol{G}^{H} \boldsymbol{H}^{H}\right| .
$$

Therefore, the optimization problem is now given as:

$$
\begin{array}{ll}
\underset{\boldsymbol{A}_{R F}, \boldsymbol{D}_{B B}, N_{R F}^{t}}{\arg \max } & \gamma_{E E}=\frac{C}{P_{\text {total }}}, \\
& \text { s.t. } \quad C \geq C_{\text {min }}, \quad \operatorname{tr}\left(\boldsymbol{G} \boldsymbol{G}^{H}\right) \leq P_{\max }, \\
& \left|\left(\boldsymbol{A}_{R F}\right)_{n_{t}}\right|=1, \forall n_{t}, \\
& \|\boldsymbol{G}\|_{F}^{2}=N_{s}, \\
& 1 \leq N_{R F}^{t} \leq N_{t},
\end{array}
$$

where $C_{\min }$ is the minimum desired spectral efficiency. The non-convex constant modulus constraint is subjected to $A_{R F}$ as restricting all the non-zero elements to have the same amplitude in the analog domain. The total power consumed $P_{\text {total }}$ equals the total power consumed in 
the transmitter side $P_{\text {Ttotal }}$ in (28). The total transmit power constraint and limited number of RF chains between 1 and the maximum number of antennas are considered respectively in (32). Undoubtedly, the optimization problem in this paper targets the combination of different parameters $A_{R F}, D_{B B}$, and $N_{R F}^{t}$ simultaneously which renders this problem to be untractable. In this work, it is assumed that $A_{R F}^{o p t}, D_{B B}^{o p t}$, and $N_{R F}^{t^{o p t}}$ are the optimal solutions to the above mentioned problem. Let us first try to optimize the number of RF chains.

\section{B. Optimizing Number of RF Chains via Applying Bisection Algorithm}

The architecture in Fig. 1 shows that a digital switches block is added into the transmitter and receiver side of hybrid D-A mm-Wave system. The main role of these switches is to activate only the optimal number of RF chains in the system. These switches have to execute an order either active which means use an RF chain, or open which means no need to use the RF chain. It can be observed from (12a) that the spectral efficiency of the system is a function of $\boldsymbol{H}$ and $\boldsymbol{G}$ where $\boldsymbol{G}=\boldsymbol{A}_{R F} \boldsymbol{D}_{B B}$. Exploiting this feature leads to simplifying the implementation of optimizing the number of RF chains algorithm by targeting a spectral efficiency that does not require the precoder and combiner matrices to be implemented regularly [22]. This avoids the re-run of the hybrid D-A designing algorithms for each possible preferred of active RF chains. Therefore, the spectral efficiency problem in (31) can be reformulated as a power allocation problem by taking the SVD of $H$ and $G$ yielding [22]

$$
C\left(N_{R F}^{t},\left\{p_{i}\right\}\right)=\sum_{i=1}^{N_{R F}^{t}} \log _{2}\left(1+\frac{1}{\sigma^{2}} \lambda_{i}^{2} p_{i}\right),
$$

where $\lambda_{i}$ corresponds to the eigenvector of $G$ and $p_{i}$ denotes the power allocated to the $i$-th RF chain. The total power in (28) can be a function of this power allocation by replacing $\operatorname{tr}\left(\boldsymbol{G} \boldsymbol{G}^{H}\right)$ as

$$
\begin{aligned}
P_{\text {total }}\left(p_{i}\right) & =\beta \sum_{i=1}^{N_{R F}^{t}}\left(p_{i}+N_{R F}^{t}\left(P_{D A C}+P_{R F}\right)\right) \\
& +N_{t}\left(P_{P A}+N_{R F}^{t} P_{P S}\right)+P_{B P} .
\end{aligned}
$$

From (33) and (34) the optimization function in (32) is redesigned to properly choose the optimized number of RF chains at the transmitter $N_{R F}^{t^{o p t}}$ as 


$$
\begin{aligned}
& N_{R F}^{t^{\text {opt }}=} \underset{N_{R F}^{t}}{\arg \max } \frac{C\left(N_{R F}^{t},\left\{p_{i}\right\}\right)}{P_{\text {total }}\left(N_{R F}^{t},\left\{p_{i}\right\}\right)} \\
& \text { s.t. } \quad C\left(N_{R F}^{t},\left\{p_{i}\right\}\right) \geq C_{\min }, \\
& \sum_{i=1}^{N_{R F}^{t}} p_{i} \leq P_{\max } \\
& 1 \leq N_{R F}^{t} \leq N_{t} .
\end{aligned}
$$

The objective function in (35) denotes the $\mathrm{EE}$ that is obtained through finding the optimal number of RF chains $N_{R F}^{t^{o p t}}$. This objective function can be solved by using primal fractional optimization programming which involves maximization of a ratio of two different functions [21], [31]. (35) can be transformed using Dinkelbach's method [21], and the optimal solution for a given $\varphi$ can be achieved if and only if the function $E(\varphi)=0$ which implies $\varphi=\varphi^{o p t}$ and $\varphi^{o p t}=$ $\arg \max \frac{C}{P_{\text {total }}}$. The maximization of the ratio $\frac{C}{P_{\text {total }}}$ is mathematically identical to the maximization of the difference $C-\varphi P_{\text {total }}$ for a specific choice of the parameter $\varphi$ [31]. Thus leveraging the theoretical results in [21], [31], the problem in (35) is transformed into the following parametric programming problem

$$
\begin{aligned}
E\left(N_{R F}^{t^{*}},\left\{p_{i}^{*}\right\}, \varphi^{*}\right)=\quad & \underset{N_{R F}^{t}}{\arg \max } C\left(N_{R F}^{t^{(\alpha)}},\left\{p_{i}^{\alpha}\right\}\right)-\varphi^{\alpha} P_{\text {total }}\left(N_{R F}^{t^{(\alpha)}},\left\{p_{i}^{\alpha}\right\}\right), \\
& \text { s.t. } C\left(N_{R F}^{t},\left\{p_{i}\right\}\right) \geq C_{\text {min }}, \\
& \sum_{i=1}^{N_{R F}^{t}} p_{i} \leq P_{\max }, \\
& 1 \leq N_{R F}^{t} \leq N_{t} .
\end{aligned}
$$

where $C\left(N_{R F}^{t^{(\alpha)}},\left\{p_{i}^{\alpha}\right\}\right)$ and $P_{\text {total }}\left(N_{R F}^{t^{(\alpha)}},\left\{p_{i}^{\alpha}\right\}\right)$ are computed via (33) and (34), respectively, for $\alpha=$ $1,2, \cdots, \alpha_{\max }$, where $\alpha_{\max }$ is the number of maximum iterations. The above proposition implies that if one can find a parameter $\varphi$ such that the optimal value of the objective function in (36) is zero i.e., $E(\varphi)=0$, then this optimal solution can be directly applied to (35). However, from a mathematical point of view, the function $E(\varphi)=0$ is a monotonically decreasing function over $\varphi$, in such case solving (36) through Dinkelbach's method does not guarantee an optimal solution to (35) [21]. Therefore, BI [32], as demonstrated in Algorithm 2, performs better for this problem in finding $\varphi^{\alpha}$. The BI algorithm is a simple method for finding the roots of objective functions. In $\mathrm{BI}$, it is important to decrease the search time of the procedure which is achieved by initializing $\varphi^{\alpha}$ based on lower $\varphi_{\min }^{\alpha}$ and upper bounds $\varphi_{\max }^{\alpha}$, such that $\varphi_{\min }^{\alpha} \leq \varphi^{\alpha} \leq \varphi_{\max }^{\alpha}$. Intuitively, the

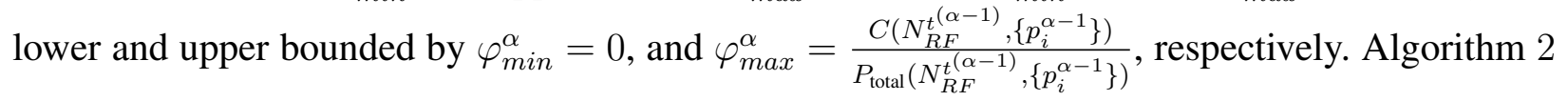




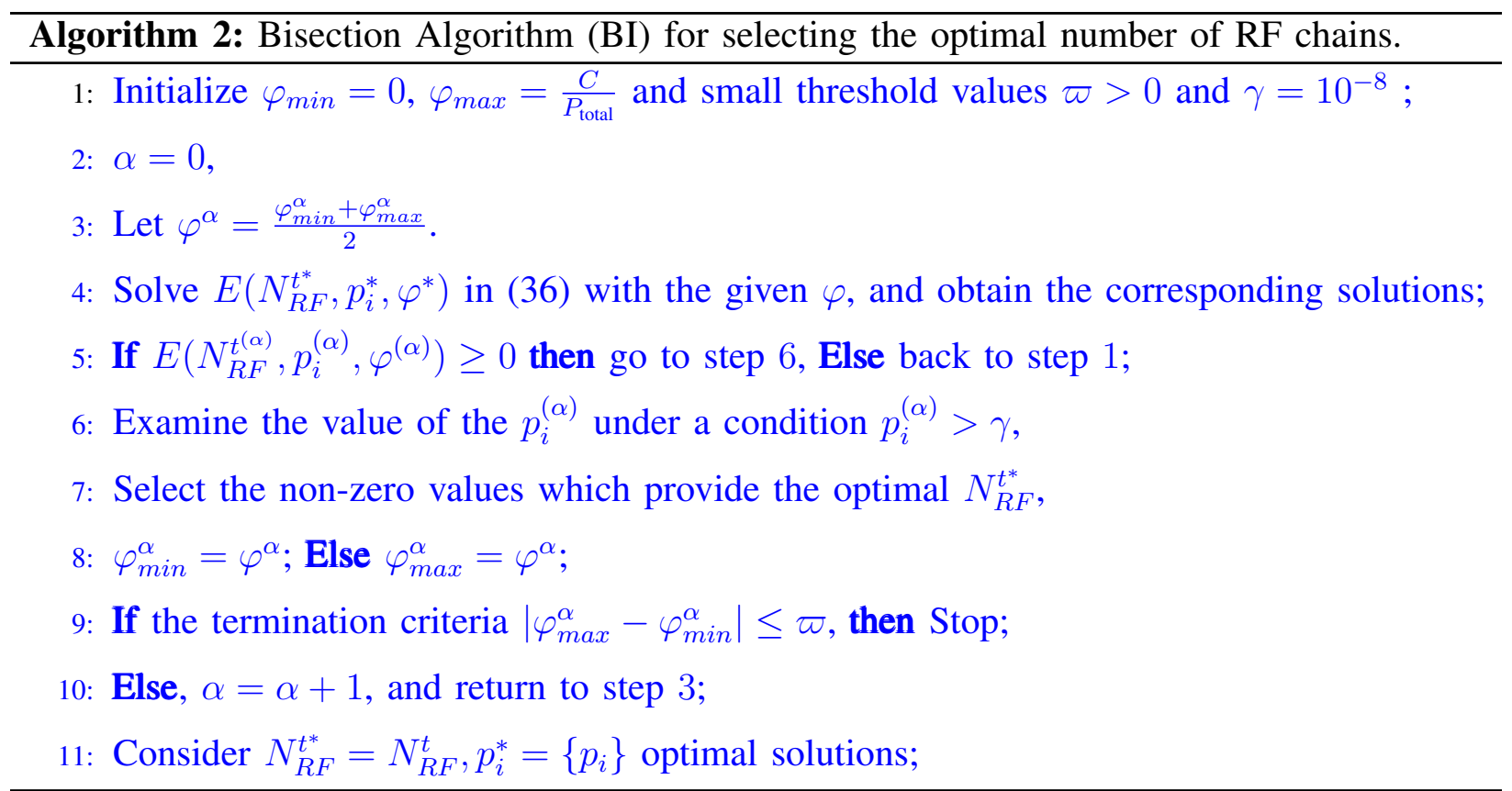

provides the BI based solution to obtain the optimal number of RF chains $N_{R F}^{t^{o p t}}$. The acquired combination of optimal power values $\left\{p_{i}^{*}\right\}$ can be represented as a power vector $\boldsymbol{p}_{\text {opt }}$. Importantly, all positive and non-zero power values are found by utilizing BI which equals the optimal number of RF chains. Essentially, when the power value equals either a negative or zero, then it is set to zero and the algorithm starts with a new RF chain until the BI finds the optimal number of RF chains for the hybrid D-A design. After calculating all the power values, the BI sends an active order to the digital switches to activate those RF chains that corresponds to a positive power value, or vice-versa sends an open order to open those RF chains that correspond to either a negative or zero value. Through the observation of the $\left\{p_{i}^{*}\right\}$ values, a threshold should be imposed because some values are significantly low, such as $10^{-8}$, or $10^{-9}$. These values cannot be considered as active powers because they cannot be detected by the system. Therefore, these values should be forced to be equal to zero and considered as an open RF chain. This results in a tolerance value $\gamma$ condition that is added in the BI in step 6. In this system model, it is assumed that the number of data streams equals the number of RF chains, therefore, this method can target the optimal number of data streams as well. Theorem 3 in [33] provides the convergence properties of BI based algorithm. Based on the analysis in [33], BI converges to the optimal $\varphi^{*}$. Any solution $\varphi$, which satisfies $\left|\varphi_{\max }-\varphi_{\min }\right| \leq \varpi$ for a small $\varpi>0$, can be found within at most $\alpha$ iterations, where $\alpha$ is the minimum integer such that $\alpha \geq \log _{2}\left(\frac{1}{\varpi} \cdot \frac{C}{P_{\text {total }}}\right)$. The BI 
algorithm that is used to optimize the number of RF chains at the transmitted side can also be applied directly to the receiver side.

An, an efficient method to optimally find one of the main parameters that this work targets to optimize, the number of RF chains, is now completed. The remaining task is to obtain the precoding D-A design which will be discussed in the next section.

\section{Vi. Computational Complexity of Proposed Hybrid D-A MM-Wave Large}

\section{ANTENNA ARRAY SySTEMS}

The complexity of the hybrid D-A mm-Wave system is calculated by taking into account the number of multiplications and additions involved as shown, in Table-I and Table-II. Since mmWave systems use sophisticated large antenna arrays, the computational complexity is dominated by the number of antennas. The complexity of the proposed EVD method for designing the analog precoder consists of two stages: multiplying two-channel matrices and then decomposing the desired matrix $\mathbf{P}_{T}$. It requires $N_{t}^{3}$ multiplications and $N_{t}\left(N_{r}-1\right)+N_{t}\left(N_{t}-1\right)$ additions. The inverse of a matrix for any algorithm is derived with the help of Cholesky decomposition. Notice that, the number of antennas involved in calculating the computational complexity of the proposed EVD method has a smaller impact on the system complexity compared to the MO-AltMin and OMP algorithms as shown, in Table-I. Moreover, the proposed PI algorithm only requires $N_{t}^{2} N_{R F}^{t}$ multiplications and $N_{t}\left(N_{r}-1\right)+N_{t}\left(N_{t}-1\right)$ additions, with which it achieves the lowest computational complexity. The number of iterations has a clear impact on the overall complexity of iterative algorithms. In Table-I, the calculation of the computational complexity per iteration is listed. The overall computational complexity of each algorithm needs to be multiplied by the required number of iterations for each algorithm. Suppose that the hybrid D-A mm-Wave system is designed based on $N_{R F}^{t}=N_{s}=2, N_{t}=144, N_{r}=64$, and $L=10$, where $L$ is the number multi-path, which yields the following computational complexity for MO-AltMin, OMP and the proposed methods:

1) In the OMP algorithm, the number of multiplications to complete the first iteration is 119, 439, 360. In general, OMP needs approximately 5 to 10 iterations to converge, resulting in a minimum number of multiplications of $597,196,800$.

2) The MO-AltMin algorithm requires 456, 480 number of multiplications to complete the first iteration. However, it needs 20 iterations to converge which results in a total number of multiplications of 9, 129,600 in order to design the analog precoder in the system [14]. 
Table I: Computational Complexity of Analog Precoders.

\begin{tabular}{|l|l|l|}
\hline \multirow{2}{*}{\multicolumn{1}{|c|}{ Algorithm }} & \multicolumn{2}{|c|}{ Number of operations per iteration } \\
\cline { 2 - 3 } & \multicolumn{1}{|c|}{ Additions } & \multicolumn{1}{c|}{ Multiplications } \\
\hline MO-AltMin analog precoding [14] & $\begin{array}{c}2 N_{s}\left(N_{t} N_{R F}^{t}-1\right) N_{t}+N_{t} N_{s}\left(N_{t} N_{R F}^{t}-\right. \\
1)+N_{t} N_{R F}^{t}\left(N_{t} N_{s}-1\right)+ \\
2 N_{t} N_{R F}^{t}\left(N_{t} N_{R F}^{t}-1\right)\end{array}$ & $\begin{array}{l}2 N_{R F}^{t} N_{s} N_{t}^{2}+N_{t}^{2} N_{s} N_{R F}^{t}+N_{t}^{2} N_{s}+ \\
2 N_{t}^{2} N_{R F}^{t^{2}}+N_{t} N_{R F}^{t}\end{array}$ \\
\hline OMP analog precoding [18] & $\frac{N_{R F}^{t^{3}}+N_{R F}^{t}\left(N_{t}-1\right) L N_{s}}{6}$ & $N_{s} N_{R F}^{t} N_{t}^{3} L$ \\
\hline GP analog precoding [20] & $\frac{N_{s}^{3}}{6}+N_{t}\left(N_{R F}^{t}-1\right) N s$ & $N_{s} N_{R F}^{t}{ }^{2} N_{t}^{2}$ \\
\hline $\begin{array}{l}\text { Proposed analog precoding based } \\
\text { on EVD scheme }\end{array}$ & $N_{t}\left(N_{r}-1\right)+N_{t}\left(N_{t}-1\right)$ & $N_{t}^{3}$ \\
\hline $\begin{array}{l}\text { Proposed analog precoding based } \\
\text { on PI algorithm }\end{array}$ & $N_{t}\left(N_{r}-1\right)+N_{t}\left(N_{t}-1\right)$ & $N_{t}^{2} N_{R F}^{t}$ \\
\hline
\end{tabular}

3) The GP algorithm requires 165,888 multiplications per iteration and it needs 4 iterations to converge which results in a total number of multiplications of 663,552 to design the analog precoder in the system [20].

4) The proposed EVD method does not require an iterative procedure. In this case, to design the analog precoder in the system, the number of multiplications is resulting in a minimum number of multiplications of $2,985,984$.

5) The proposed PI iterative algorithm needs 41,472 multiplications to complete the first iteration. Until PI converges and completes designing the analog precoder, it requires only 4 iterations which makes the total number of multiplications 207, 360 .

Undoubtedly, the number of multiplications that is demanded to perform the proposed methods is significantly lower than that of the OMP, MO-AltMin, and GP algorithms. Note here, that in the previous calculations the number of multiplications is only considered because the multiplication operations consume more computations than additions [34].

For designing the digital precoder, the proposed method based on SVD consists of three stages. Therefore, it requires $N_{R F}^{t} N_{r}\left(N_{t}-1\right)+N_{R F}^{t}\left(N_{r}-1\right)$ additions and $N_{R F}^{t} N_{r} N_{t}+N_{R F}^{t^{2}} N_{r}+N_{R F}^{t^{3}}$ multiplications. It can be noticed that the number of RF chains has a large impact on the computational complexity of the proposed method. Essentially in the hybrid D-A mm-Wave systems the number of RF chains is roughly small as compared to the number of antennas, as result, the attained complexity of the proposed method is lower than that of the LS solution. For the same numerical example, the number of multiplications that are required in the proposed 
Table II: Computational Complexity of Digital Precoders.

\begin{tabular}{|l|l|l|}
\hline \multirow{2}{*}{ Algorithm } & \multicolumn{1}{|c|}{ Number of operations per iterations } \\
\cline { 2 - 3 } & \multicolumn{1}{|c|}{ Additions } & \multicolumn{1}{|c|}{ Multiplications } \\
\hline $\begin{array}{l}\text { LS solution for digital precoding in } \\
\text { [14], [18], and [20] }\end{array}$ & $\begin{array}{l}N_{R F}^{t}\left(N_{t}-1\right)+N_{r}\left(N_{t}-1\right)+ \\
N_{R F}^{t} N_{r}\left(N_{t}^{2}-1\right)\end{array}$ & $N_{R F}^{t} N_{t}^{2}+N_{s} N_{t}^{2}+N_{R F}^{t} N_{t} N_{s}$ \\
\hline $\begin{array}{l}\text { Proposed digital precoding based } \\
\text { on SVD }\end{array}$ & $N_{R F}^{t} N_{r}\left(N_{t}-1\right)+N_{R F}^{t}\left(N_{r}-1\right)$ & $N_{R F}^{t} N_{r} N_{t}+N_{R F}^{t^{2}} N_{r}+N_{R F}^{t^{3}}$ \\
\hline
\end{tabular}

method based on an effective channel is 18,696 and in the conventional LS solution, it is 83,520 , respectively.

To summarize, the proposed methods for the analog and digital precoders are preferred over the existing algorithms, due to their low complexity as shown in the simulation result Section V.

Finally, after obtaining $A_{R F}^{F^{o p t H}}(\hat{\vartheta}), D_{B B}^{o p t}, N_{R F}^{t^{o p t}}$, and $\boldsymbol{p}_{o p t}$, the joint precoder is designed as $\boldsymbol{G}^{o p t}=\boldsymbol{A}_{R F}^{F^{o p t} H}(\hat{\vartheta}) \boldsymbol{D}_{B B}^{o p t} \operatorname{diag}\left[\boldsymbol{p}_{\text {opt }}\right]^{\frac{1}{2}}$. Now, the spectral efficiency is determined by (31), the total power by (28) and the EE by (27).

\section{Simulation Results}

In this section, the EE performance evaluation of the desired hybrid precoding system is carried out. The algorithms performances are compared with several existing algorithms. The first existing algorithm is based on beam steering which utilizes a single RF chain as proposed in [17]. The second algorithm OMP [18], third algorithm MO-AltMin [14] and the fourth system based on the combination of DM+GP algorithms [20] are also simulated with a fixed and dynamic number RF chains. The fully digital system is applied as a benchmark in which the number of RF chains is equal to the number of antennas. The mm-Wave channel as described in Section II is deployed and the number of multipaths is set to $L=15$ [14], [18]. The transmitter antenna array is assumed to be a UPA with the antenna spacing $u_{q}=\frac{\lambda}{2}$. The AoAs and AoDs are generated independently from the uniform distribution with the mean cluster angle uniformly distributed over $[0,2 \pi]$, while the angle spread is set to 7.5 [14], [18], [26]. Moreover, the minimum spectral efficiency is fixed as $C_{\min }=1 \mathrm{bits} / \mathrm{s} / \mathrm{Hz}$. Table III provides the practical values of the power parameters that are used in the simulation results [13], [14], [19] and [30].

Fig. 3 illustrates the effect on the EE of the number of transmit antennas when the SNR is fixed to $15 \mathrm{~dB}$. In the simulation, the existing hybrid D-A precoding algorithms OMP and MOAltMin utilize $6 \mathrm{RF}$ chains. It is observed that the proposed hybrid precoding based on EVD and 
Table III: The power expressions of different precoding systems where used in the simulation results [13], [14], [19], [28] and [30].

\begin{tabular}{|l|l|}
\hline \multicolumn{1}{|c|}{ Power Type } & Values \\
\hline$P_{\text {mixer }}$ & $19 \mathrm{~mW}$ \\
\hline$P_{L O}$ & $5 \mathrm{~mW}$ \\
\hline$P_{\text {filter }}$ & $14 \mathrm{~mW}$ \\
\hline$P_{B A}$ & $5 \mathrm{~mW}$ \\
\hline$P_{P S}$ & $10 \mathrm{~mW}$ \\
\hline$P_{D A C}=P_{B P}$ & $255 \mathrm{~mW}$ \\
\hline$P_{L N A}$ & $39 \mathrm{~mW}$ \\
\hline$P_{S P}$ & $19.5 \mathrm{~mW}$ \\
\hline
\end{tabular}

PI attain superior EE as compared to the other known solutions in the literature. Moreover, an increasing number of transmit antennas linearly decreases the EE of the known solutions but not for the proposed ones. The combination of the proposed BI solution with the proposed hybrid precoding algorithms enhance the EE by searching the optimal number of RF chains and then implementing the optimal D-A precoders in the system. In the fully digital system the number of transmit antennas is equal to the number of RF chains, therefore, because the number of antennas increases, the EE performance decreases as the RF chain consumes more power. Due to the lack of optimizing the number of RF chains, the DM+GP algorithms achieve lower gains than the proposed algorithms. For both the OMP and MO-AltMin hybrid precoding the EE is decreasing linearly because the number of RF chains has not been optimized. The simplified beam steering neither achieves the highest nor the lowest EE because it does not include an optimization of the number of the transmitter antennas.

Fig. 4 shows the EE variation for different precoding solutions concerning SNR. For the $64 \times 16 \mathrm{~mm}$-Wave system, it is very clear that the proposed EE hybrid precoding system based on the proposed algorithms outperforms the existing precoding solutions in [14], [17], [18] and [20] with an increase in SNR. This is due to the design of the proposed hybrid precoding being based on finding the optimal number of RF chains and then applying the optimal D-A precoders. The hybrid precoding based on OMP and MO-AltMin algorithms achieve a reasonable EE performance because their main focus is to find only the optimal D-A precoders in the system. Finally, both the fully digital and the beam steering system have the lowest performance as compared to the hybrid D-A precoding system. 




Figure 3: EE performance versus the number of transmitter antennas for $\mathrm{SNR}=15 \mathrm{~dB}$.

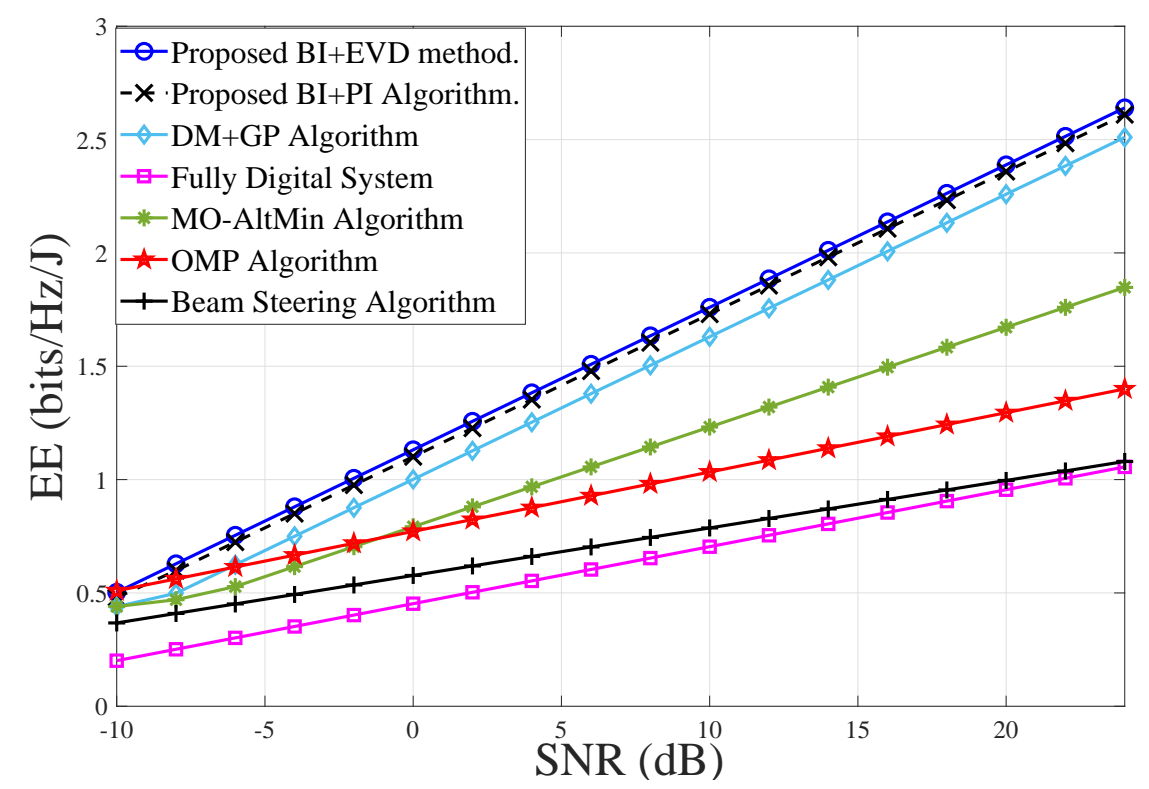

Figure 4: EE performance versus SNR when $N_{t}=64 \times N_{r}=16, \mathrm{~B}=\infty$.

In Fig. 5 the $\mathrm{EE}$ of the different algorithms based on different systems with respect to the transmission power is displayed for $N_{t}=64$ and $N_{r}=48$, and the number of RF chains is fixed as $N_{R F}^{t}=N_{s}=4$ for the MO-AltMin [14], and the OMP [18] based hybrid D-A precoding systems. Since both the proposed algorithms and DM+GP algorithms are based on searching the 


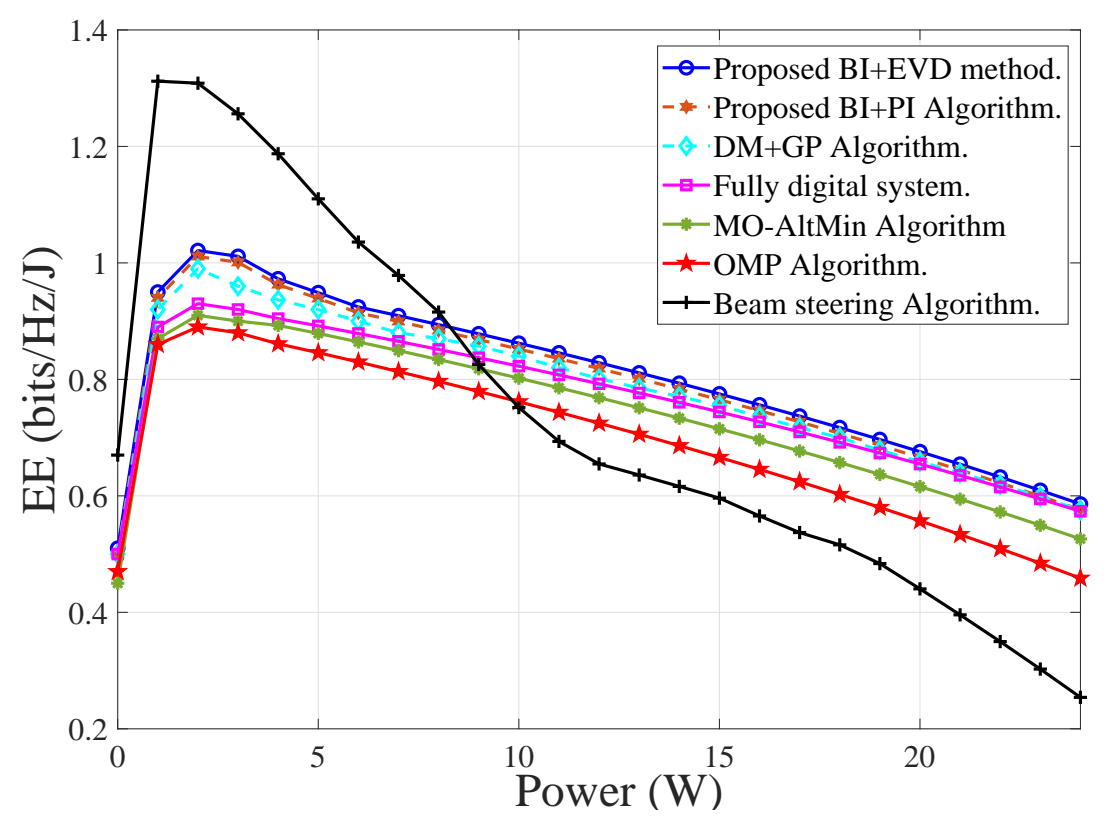

Figure 5: EE performance versus transmission power when $N_{t}=64 \times N_{r}=48$, with fixed RF chains $N_{R F}^{t}=$ $N_{s}=4, \mathrm{~B}=\infty$.

optimal number of RF chains in the system, the number of RF chains will be dynamic. When the transmission power is higher than $8 \mathrm{~W}$, the proposed algorithms are more energy efficient than the existing algorithms. For transmission up to $8 \mathrm{~W}$, the beam steering algorithm which only has a single RF chain, has the highest EE, as the gain in spectral efficiency is slowly increasing with power. However, as the power increases above that value, the beam steering performance decreases sharply as the spectral efficiency deteriorates with power consumption. Furthermore, the MO-AltMin and OMP algorithms achieve lower EE than that of those proposed algorithms, since both algorithms focus on the precoding solutions only, without any efforts to optimize the total power of the hybrid D-A system which increases the impact of the power costs on their EE. Moreover, since the OMP algorithm is based on codebook, the analog precoder performance is limited, which directly affects the gain of spectral efficiency as compared to the power costs. On the other hand, the proposed algorithms always achieve superior EE, as the spectral efficiency is close to the fully digital system and the total power is optimized which balances the EE performance. In general, with growing power consumption all algorithms, display a linearly decreasing EE since the achievable capacity has a lower EE gain compared to the cost of increased power consumption.

Fig. 6 plots the spectral efficiency performance for the hybrid D-A mm-Wave system as a 


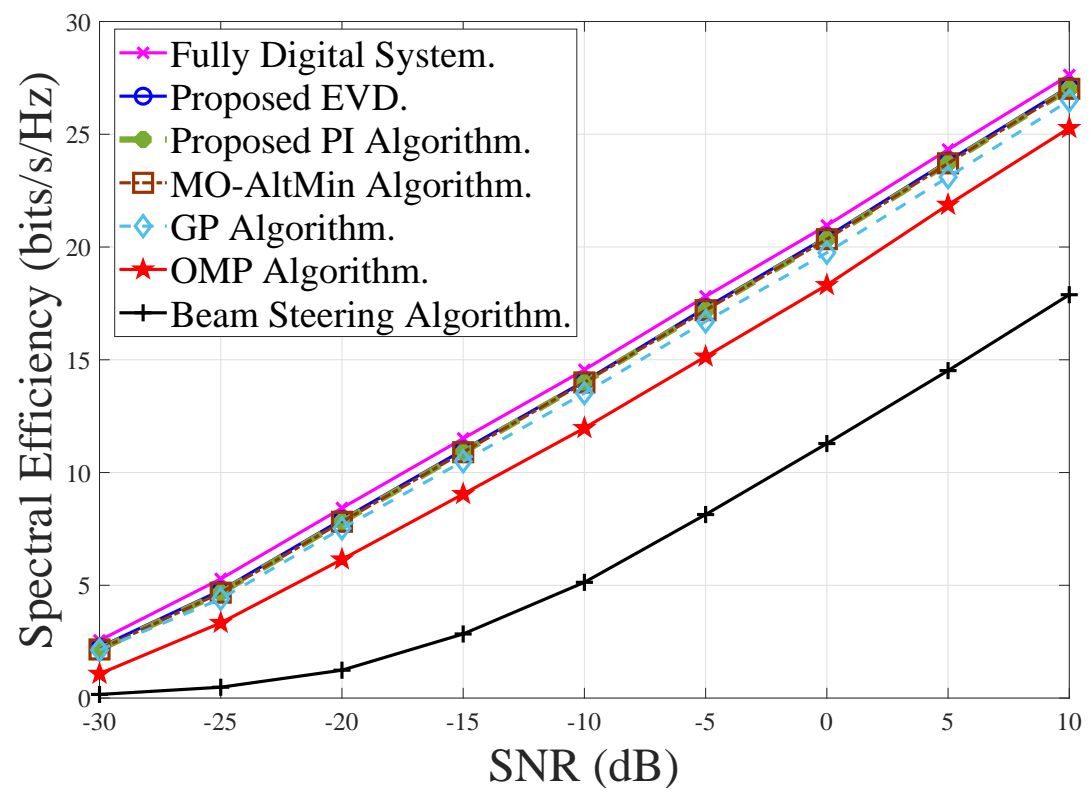

Figure 6: Spectral efficiency of hybrid D-A mm-Wave systems versus SNR when various algorithms are used and $N_{R F}^{t}=N_{R F}^{r}=2, N_{t}=128, N_{r}=32, \mathrm{~B}=\infty$.

function of SNR when $N_{R F}^{t}=N_{R F}^{r}=N_{s}$. Since the beam steering in [17] employs a single $\mathrm{RF}$ chain, the spectral efficiency achieved by beam steering is always lower than the spectral efficiency achieved by the proposed algorithms and the benchmark which is the fully digital system. OMP [18] is designed to achieve a spectral efficiency very close to the fully digital system only for a special scenario when $N_{R F}^{t} \geq 2 N_{s}$. Therefore, when the case $N_{R F}^{t}=N_{R F}^{r}=$ $N_{s}$ is applied, the spectral efficiency performance is not close to the fully digital system. It is observed that the spectral efficiency obtained by the proposed hybrid precoder based on, firstly, EVD and LS, secondly, PI and LS methods outperform the OMP and the beam steering and is slightly higher than the MO-AltMin performance, which is close to the fully digital system. The reason is that the proposed precoding methods can accurately design the optimal precoders which maximizes the spectral efficiency of the system and therefore, outperforms the existing algorithms. For the proposed precoder based on PI and LS methods, the obtained spectral efficiency performance is the same as for MO-AltMin, which is close to the optimal fully digital system.

Fig. 7 presents the spectral efficiency and the EE concerning the number of quantized bits of the resolution of the PSs. Initially, as expected, the spectral efficiency of the finite-bit grows with resolution. It can be observed that the proposed hybrid precoding based on the EVD-method 


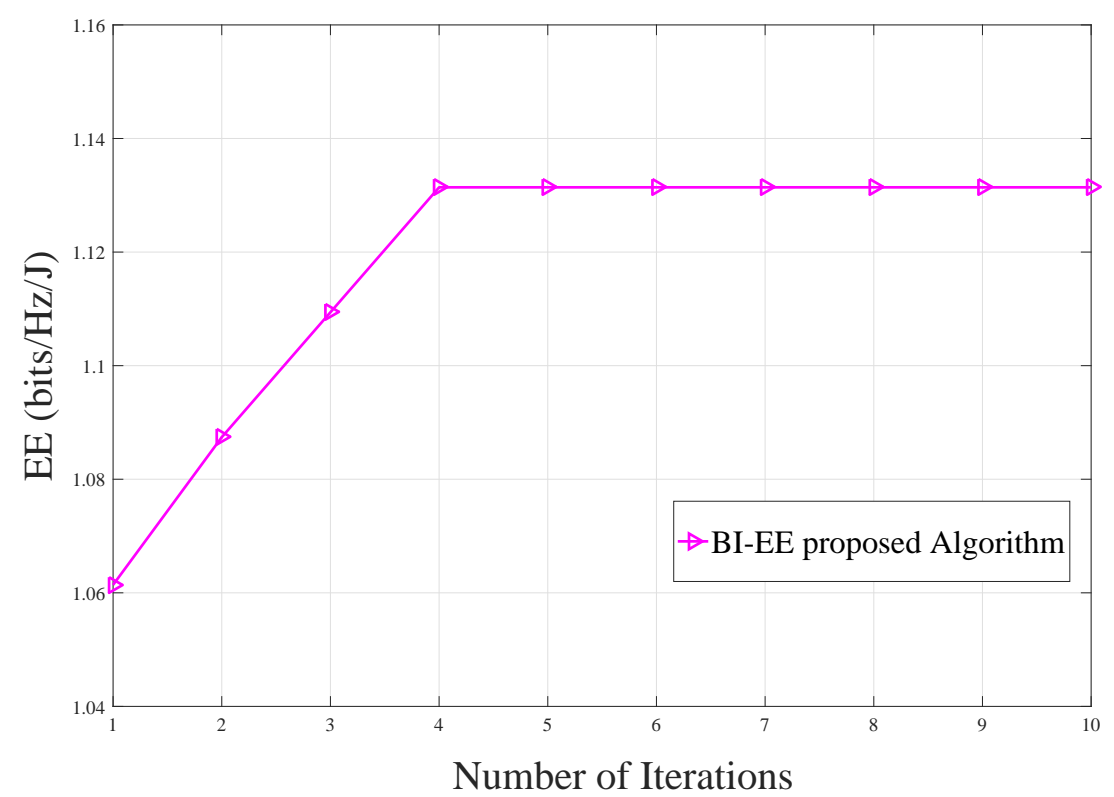

Figure 8: EE-Convergence with iteration number of the proposed BI algorithm.

achieves a similar performance for $\mathrm{B}=8$ bits as for $\mathrm{B}=\infty$. This implies that increasing the number of bits of precision does not always provide much gain as compared to for few-finitebits. This provides insight into the optimal number of quantization bits required for the proposed hybrid precoding algorithms. From the presented simulation results it can be observed that when 


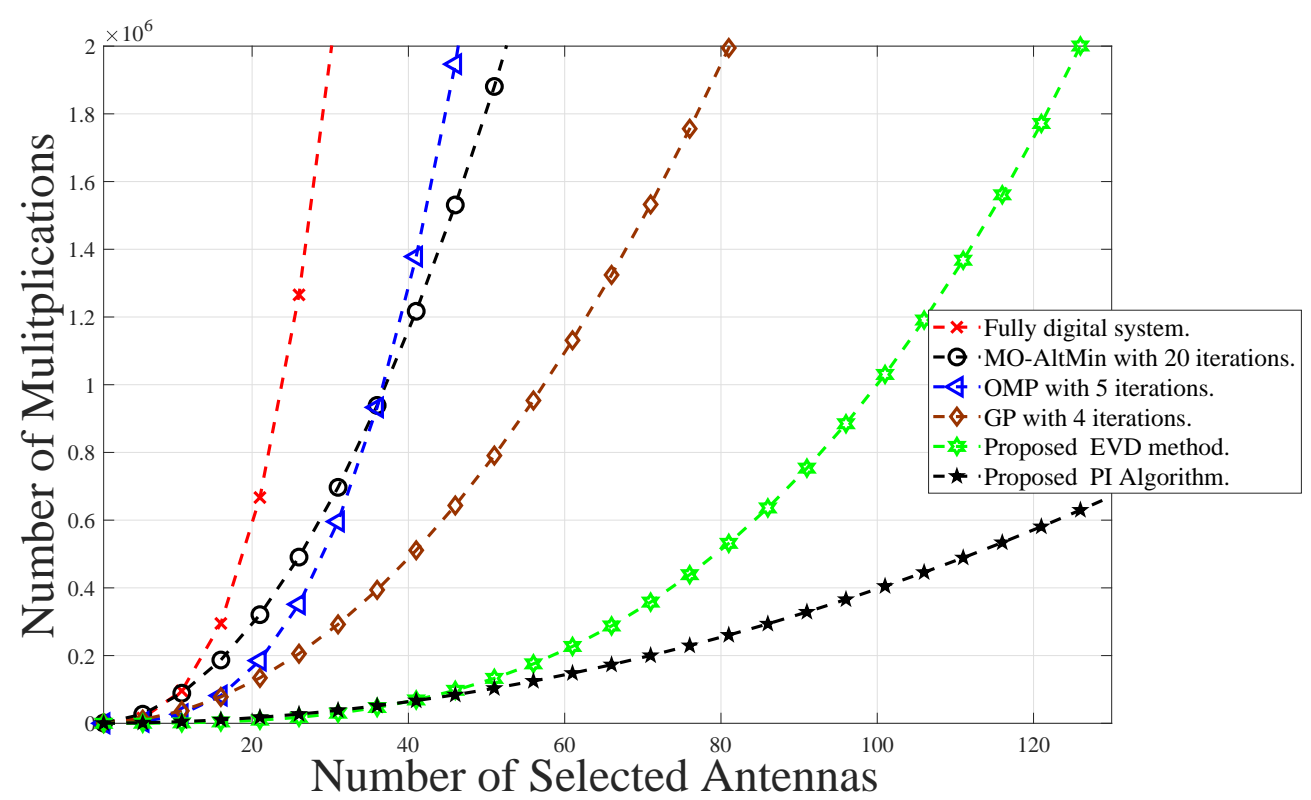

Figure 9: Number of multiplications versus the number of selected antennas for Analog precoder complexities.

the quantization $\mathrm{B}=4-5$ bits, the maximum $\mathrm{EE}$ is achieved. This is because the hybrid precoding at very-low resolution $(\mathrm{B}=1-2$ bits) suffers from severe lack of spectral efficiency, while at high resolution ( $\mathrm{B}=7-8$ bits) the power consumption increases linearly.

Fig. 8 shows the convergence behavior of the BI algorithm that searches for the optimal number of RF chains when SNR $=0 \mathrm{~dB}$. The spectral efficiency achieved is averaged over 10, 000 independent channel generations. It can be observed that BI algorithm converges very fast and only 4 iterations are required. The reason for fast convergence is that the BI has a very simple structure and is bounded to find the solutions quickly.

In both Fig. 9 and Fig. 10 the structure of the hybrid D-A mm-Wave system is based on $N_{R F}^{t}=N_{s}=2$, and $N_{r}=16$. Fig. 9 plots the number of multiplications for designing the analog precoder for the hybrid D-A mm-Wave system with respect to the number of antennas, as multiplications have a higher computational consumption than additions. The fully digital system has a high complexity because the number of RF chains is proportional to the number of antennas. From the figure, it is observed that the complexity of OMP with 5 iterations increases exponentially. The complexity of MO-AltMin with 20 iterations is lower than that of OMP when the number of antennas increases, whereas, the design of the GP algorithm is reducing the complexity of the aforementioned algorithms. However, the proposed scheme based on EVD 


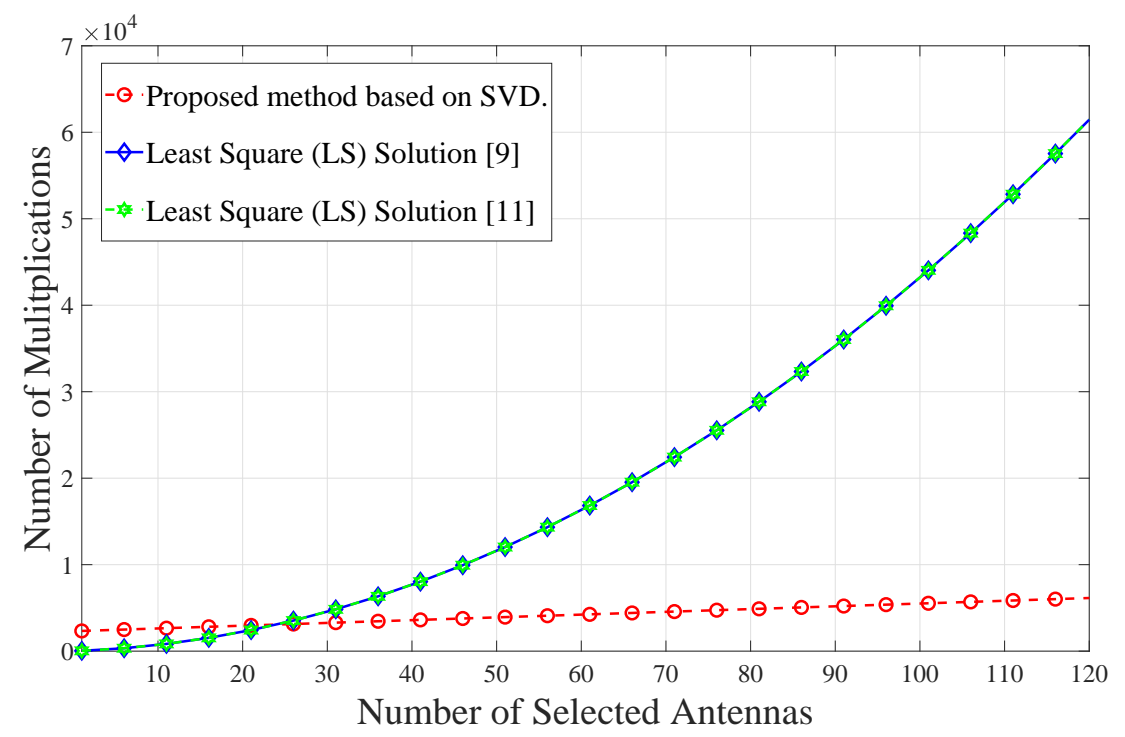

Figure 10: Number of multiplications versus the number of selected antennas for Digital precoder complexities.

increases slowly with the number of antennas. Ultimately, the analog precoder based on PI attains the least complex. It is also verified that by increasing the number of antennas, the complexity of the system increases. Therefore, in the analog precoder that is designed for the hybrid D-A mm-Wave system, the proposed scheme based on PI algorithm has the lowest complexity of all algorithms, including MO-AltMin, OMP and GP.

Fig. 10 demonstrates the computational complexity for designing the digital precoder of the hybrid D-A mm-Wave system as a function of the number of multiplications versus the number of antennas. It is observed that the complexity of the LS solution grows exponentially because when calculating the computational complexity of the LS solution the number of antennas involved in the calculation is doubled, as shown in Table II. On the hand, the complexity of the proposed scheme is based on the SVD, which increases linearly. It is shown that at a very low number of antennas $(<22)$ the proposed scheme has a slightly higher complexity than the LS solution. This is because the proposed method is designed based on the effective channel $H^{E F}$ as mentioned in Section-IV.C which involves the number of receiver antennas in the calculation. However, as the number of receiving antennas kept constant and the number of transmitter antennas increases ( $>22)$ the computational complexity of the proposed method obtains a much lower complexity than the LS solution. Therefore, in the digital precoder that is designed for the hybrid D-A system, the proposed scheme based on SVD and the effective channel has the lowest complexity 
compared to the conventional LS solution.

\section{CONCLUSIONS}

In this paper an EE hybrid precoding mm-Wave system was designed. The optimization problem was separately optimized. Firstly, the number of RF chains was optimized in the system for which the BI algorithm was proposed. This algorithm also optimized the number of data streams in the system. Secondly, the optimal analog precoder was designed with the help of the EVD method without the need for finding the gradient of the cost function. To further lower the complexity of the hybrid precoding, the PI algorithm for designing the analog precoder was proposed, whereas the digital precoder was designed based on the LS solution. Finally, the proposed hybrid precoding was based on the limited resolution of phase shifters. Potential EE gain compared to hybrid precoding with infinite bits of resolution was observed. This showed that neither does increasing the number of quantized bits of the resolution results in more spectral efficiency nor do a few-finite-bits (i.e., 1-2 bits) achieve the best EE for the hybrid precoding systems. Simulation results displayed significant EE performance advantages of the proposed algorithms over existing systems, such as the fully digital system, the MO-AltMin, OMP and DM+GP algorithms based on hybrid precoding, and the beam steering system based on a single RF chain.

\section{REFERENCES}

[1] I. Keshavarzian, Z. Zeinalpour-Yazdi, and A. Tadaion, "Energy-Efficient Mobility-Aware Caching Algorithms for Clustered Small Cells in Ultra-Dense Networks," IEEE Transactions on Vehicular Technology, vol. 68, no. 7, pp. 6833-6846, July 2019.

[2] S. Wang, R. Ruby, V. C. M. Leung, and Z. Yao, "A Low-Complexity Power Allocation Strategy to Minimize Sum-SourcePower for Multi-User Single-AF-Relay Networks," IEEE Transactions on Communications, vol. 64, no. 8, pp. 3275-3283, Aug 2016.

[3] O. Alluhaibi, E. Kampert, P. A. Jennings, and M. D. Higgins, "Impact of Overlapped AoAs on the Achievable Uplink Rate of Hybrid Beamforming for Massive MIMO mm-Wave Systems for Industrial Environments," IEEE Access, vol. 7, pp. $101178-101194,2019$.

[4] M. Naeem, A. Anpalagan, M. Jaseemuddin, and D. C. Lee, "Resource Allocation Techniques in Cooperative Cognitive Radio Networks," IEEE Communications Surveys Tutorials, vol. 16, no. 2, pp. 729-744, Second 2014.

[5] J. Opadere, Q. Liu, T. Han, and N. Ansari, "Energy-Efficient Virtual Radio Access Networks for Multi-Operators Cooperative Cellular Networks," IEEE Transactions on Green Communications and Networking, vol. 3, no. 3, pp. 603-614, Sep. 2019.

[6] Cisco Visual Networking, “Global Mobile Data Traffic Forecast Update, 2017-2022,” Feb. 2017. [Online]. Available: http://web.archive.org/web/20080207010024/http://www.808multimedia.com/winnt/kernel.htm

[7] T. S. Rappaport, G. R. MacCartney, M. K. Samimi, and S. Sun, "Wideband Millimeter-Wave Propagation Measurements and Channel Models for Future Wireless Communication System Design," IEEE Trans. on Commun., vol. 63, no. 9, pp. 3029-3056, Sep. 2015.

[8] B. Yang, Z. Yu, J. Lan, R. Zhang, J. Zhou, and W. Hong, "Digital Beamforming-Based Massive MIMO Transceiver for 5G Millimeter-Wave Communications," IEEE Transactions on Microwave Theory and Techniques, vol. 66, no. 7, pp. 3403-3418, July 2018.

[9] A. W. Mbugua, W. Fan, Y. Ji, and G. F. Pedersen, "Millimeter Wave Multi-User Performance Evaluation Based on Measured Channels With Virtual Antenna Array Channel Sounder," IEEE Access, vol. 6, pp. 12 318-12 326, 2018. 
[10] M. Alonzo, S. Buzzi, A. Zappone, and C. D’Elia, "Energy-Efficient Power Control in Cell-Free and User-Centric Massive MIMO at Millimeter Wave," IEEE Transactions on Green Communications and Networking, vol. 3, no. 3, pp. 651-663, Sep. 2019.

[11] C. Rusu, R. Mèndez-Rial, N. González-Prelcic, and R. W. Heath, "Low Complexity Hybrid Precoding Strategies for Millimeter Wave Communication Systems," IEEE Transactions on Wireless Communications, vol. 15, no. 12, pp. 83808393, Dec 2016.

[12] R. Méndez-Rial, C. Rusu, N. González-Prelcic, and R. W. Heath, "Dictionary-free hybrid precoders and combiners for mmWave MIMO systems," in 2015 IEEE 16th International Workshop on Signal Processing Advances in Wireless Communications (SPAWC), June 2015, pp. 151-155.

[13] X. Gao, L. Dai, S. Han, C. L. I, and R. W. Heath, "Energy-Efficient Hybrid Analog and Digital Precoding for MmWave MIMO Systems With Large Antenna Arrays," IEEE Jour.l on Sel. Areas in Commui., vol. 34, no. 4, pp. 998-1009, Apr. 2016.

[14] X. Yu, J. C. Shen, J. Zhang, and K. B. Letaief, "Alternating Minimization Algorithms for Hybrid Precoding in Millimeter Wave MIMO Systems," IEEE J. Sel. Topics in Signal Processing, vol. 10, no. 3, pp. 485-500, Apr. 2016.

[15] S. Buzzi, C. I, T. E. Klein, H. V. Poor, C. Yang, and A. Zappone, "A Survey of Energy-Efficient Techniques for 5G Networks and Challenges Ahead," IEEE Journal on Selected Areas in Communications, vol. 34, no. 4, pp. 697-709, April 2016.

[16] S. Hur, S. Baek, B. Kim, Y. Chang, A. F. Molisch, T. S. Rappaport, K. Haneda, and J. Park, "Proposal on Millimeter-Wave Channel Modeling for 5G Cellular System," IEEE J. Sel. Areas in Signal Processing, vol. 10, no. 3, pp. 454-469, April 2016.

[17] O. E. Ayach, R. W. Heath, S. Abu-Surra, S. Rajagopal, and Z. Pi, "The capacity optimality of beam steering in large millimeter wave MIMO systems," in IEEE 13th International Workshop on Signal Processing Advances in Wireless Commun. (SPAWC), Jun. 2012, pp. 100-104.

[18] O. El Ayach, S. Rajagopal, S. Abu-Surra, Z. Pi, and R. W. Heath Jr., "Spatially sparse precoding in millimeter wave MIMO systems," IEEE Trans. on Wireless Commun., vol. 13, no. 3, pp. 1499-1513, Mar. 2014.

[19] R. Zi, X. Ge, J. Thompson, C. Wang, H. Wang, and T. Han, "Energy Efficiency Optimization of 5G Radio Frequency Chain Systems," IEEE Journal on Selected Areas in Communications, vol. 34, no. 4, pp. 758-771, April 2016.

[20] A. Kaushik, J. Thompson, E. Vlachos, C. Tsinos, and S. Chatzinotas, "Dynamic RF Chain Selection for Energy Efficient and Low Complexity Hybrid Beamforming in Millimeter Wave MIMO Systems," IEEE Transactions on Green Communications and Networking, pp. 1-1, 2019.

[21] I. Stancu-Minasian, Fractional Programming: Theory, Methods and Applications. Springer Netherlands, 1997.

[22] A. Goldsmith, Wireless Communications. New York, NY, USA: Cambridge University Press, 2005.

[23] L. Dai, X. Gao, J. Quan, S. Han, and C.-L. I, "Near-optimal hybrid analog and digital precoding for downlink mmWave massive MIMO systems," in Proc. IEEE ICC, Jun. 2015, pp. 1334-1339.

[24] E. Kampert, P. A. Jennings, and M. D. Higgins, "Investigating the V2V Millimeter-Wave Channel Near a Vehicular Headlight in an Engine Bay," IEEE Communications Letters, vol. 22, no. 7, pp. 1506-1509, July 2018.

[25] A. Kammoun, H. Khanfir, Z. Altman, M. Debbah, and M. Kamoun, "Preliminary results on 3D channel modeling: from theory to standardization," IEEE J. Sel. Areas in Commun., vol. 32, no. 6, pp. 1219-1229, Jun. 2014.

[26] 3rd Generation Partnership Project (3GPP), "Study on channel model for frequencies from 0.5 to 100 GHz,” Tech. Rep. 36.331, 2017, version 38.901. [Online]. Available: https://portal.3gpp.org/desktopmodules/Specifications/SpecificationDetails.aspx?specificationId=3173

[27] A. N. Langville and C. D. Meyer, Google's PageRank and Beyond: The Science of Search Engine Rankings. Princeton, NJ, USA: Princeton University Press, 2012.

[28] W. B. Abbas, F. Gomez-Cuba, and M. Zorzi, "Millimeter Wave Receiver Efficiency: A Comprehensive Comparison of Beamforming Schemes With Low Resolution ADCs," IEEE Trans. on Wireless Comm., vol. 16, no. 12, pp. 8131-8146, Dec 2017.

[29] C. Masouros, M. Sellathurai, and T. Ratnarajah, "Computationally Efficient Vector Perturbation Precoding Using Thresholded Optimization,” IEEE Trans. on Commun., vol. 61, no. 5, pp. 1880-1890, May. 2013.

[30] S. Han, C.-L. I., Z. Xu, and S. Wang, "Reference Signals Design for Hybrid Analog and Digital Beamforming," IEEE Commun. Lett., vol. 18, no. 7, pp. 1191-1193, Jul. 2014.

[31] H. P. Benson, "Fractional programming with convex quadratic forms and functions," European Journal of Operational Research, vol. 173, no. 2, pp. 351 - 369, 2006.

[32] S. Boyd and L. Vandenberghe, Convex Optimization. New York, NY, USA: Cambridge University Press, 2004.

[33] G. Miao, N. Himayat, and G. Y. Li, "Energy-efficient link adaptation in frequency-selective channels," IEEE Trans. on Commun., vol. 58, no. 2, pp. 545-554, Feb. 2010.

[34] Q. Z. Ahmed, K. H. Park, M. S. Alouini, and S. Aissa, "Linear Transceiver Design for Nonorthogonal Amplify-andForward Protocol Using a Bit Error Rate Criterion," IEEE Trans. on Wireless Commun., vol. 13, no. 4, pp. 1844-1853, Apr. 2014. 


\section{Authors' Response to Comments by}

\section{Editor's Comments}

Thank you very much for coordinating the review of our manuscript and for providing us with many useful comments from you and the reviewers. We are glad to learn that you and the reviewers deem our work is timely, novel, and the paper is generally well written. In general, the reviewers 'most comments are very helpful for improving our work. Based on their comments, we have thoroughly revised our paper and all major issues are believed to have been xed. Given its substantial improvements, we sincerely hope that you and the reviewers could reevaluate our work for a possible publication in the journal. For your convenience, the key changes made in the revision are highlighted in blue in the manuscript.

\section{Reviewer 1 Comments}

We appreciate your effort spent on carefully reviewing our manuscript and your useful feedback. Based on your comments, we have thoroughly revised our manuscript. Our point-to-point responses to your comments are given below. Please note that the references or equations mentioned below are rearranged and their numbering may differ from those in the manuscript.

1) Does the paper represent a technical contribution and, if so, what? Yes. This paper develops new algorithms that can design energy efficient hybrid D-A mm-Wave systems, in which the number of RF chains is optimized while simultaneously low complexity D-A precoders and combiners are designed.

Response: We appreciate your positive and constructive comments on our work. In particular, we are glad to learn that you deem our work having sufficient technical contributions.

2) Is the paper well written? If not, how can it be improved? No. There are a lot of grammar mistakes. The first sentence in the introduction, "... complexity are usually of most concern..." should be "... complexity are usually of the most concerns...". For the 28 -th line of page 1, “... to be deployed as part of ..." should be "... to be deployed as a part of ...". The authors are recommended to check the whole paper to make it more readable.

Response: We appreciate your effort and constructive comments, particularly, we have considered all the comments. Therefore, the paper has been revised and proofread carefully.

3) Is the paper appropriately organized? If not, how should it be reorganized? Yes. 
Response: We appreciate your positive and constructive comments on our work. In particular, we are glad to learn that you deem our work being well-organized.

4) Can the paper be shortened without diminishing the quality of the paper or its results? If so, how? No.

Response: We appreciate your positive and constructive comments on our work.

5) Is it necessary that the paper be expanded? If so, where and how? No.

Response: We appreciate your positive and constructive comments on our work.

6) If the paper is not suitable in its current form, can it be modified to make it so? How?Yes.

- The authors are suggested to add more references in recent three years.

Response: Thanks for your suggestion, we have added the following references:

a) "Energy-Efficient Power Control in Cell-Free and User-Centric Massive MIMO at Millimeter Wave", [1], IEEE Transactions on Green Communications and Networking ( Volume: 3 , Issue: 3 , Sept. 2019 ).

b) "Energy-Efficient Virtual Radio Access Networks for Multi-Operators Cooperative Cellular Networks", [2], IEEE Transactions on Green Communications and Networking ( Volume: 3 , Issue: 3 , Sept. 2019 ).

c) "A Survey of Energy-Efficient Techniques for 5G Networks and Challenges Ahead", [3], IEEE Journal on Selected Areas in Communications ( Volume: 34 , Issue: 4 , April 2016 ).

d) "Energy Efficiency Evaluation of Linear Transmitters for 5G NR Wireless Waveforms", [4], IEEE Transactions on Green Communications and Networking ( Volume: 3 , Issue: 2 , June 2019 ).

e) "Impact of Overlapped AoAs on the Achievable Uplink Rate of Hybrid Beamforming for Massive MIMO mm-Wave Systems for Industrial Environments", [5], Topic: Emerging Trends, Issues and Challenges for Array Signal Processing and Its Applications in Smart City Published in: IEEE Access ( Volume: 7 , July 2019 ).

- As such, the authors are recommended to present the differences between this paper and the other existing works, especially in recent three years.

Response: Thanks for your suggestion, we have shown the difference between our work and the recent existing works such as [6], [7] and [8]. This difference is investigated in a few sections such as the introduction, computational complexity, and simulation results sections. The recent existing work is introduced in the following references:

a) "Dynamic RF Chain Selection for Energy Efficient and Low Complexity Hybrid Beamform- 
ing in Millimeter Wave MIMO Systems", [6], IEEE Transactions on Green Communications and Networking, 2019.

b) "Energy Efficiency Optimization of 5G Radio Frequency Chain Systems", [7], IEEE Journal on Selected Areas in Communications, 2016.

c) "Alternating Minimization Algorithms for Hybrid Precoding in Millimeter Wave MIMO Systems", [8], IEEE Journal on Selected Areas in Communications, 2016.

\section{- The contributions of this work need to be reorganized to make them more concise.}

Response: We are grateful to the reviewer for this comment. We have re-organized the contribution part and hopefully it is clear and meets the reviewer's requirements. The final draft is as follows:

\section{A. Methodology and Contributions}

As noted above, in the existing hybrid D-A mm-Wave systems, neither the number of RF chains was optimized to enhance EE, nor was the reduction of the computational complexity of the hybrid D-A mm-Wave system investigated. Therefore, this paper aims to develop new algorithms that can assist in the design of energy efficient hybrid D-A mm-Wave systems, in which the number of RF chains is optimized whilst simultaneously lowering the computational complexity of the D-A precoders and combiners. Hence, the main contributions of this work are:

a) Modeling: The optimization problem is formulated as the fractional programming maximization which divides the optimization problem into two sub-problems:

i) Sub-problem One: This is formulated as a power allocation problem. Bisection algorithm (BI) is proposed for it's solution. The optimal number of RF chains is equal to the number of all the positive and non-zero power allocation values that are calculated via BI. To activate only the optimal RF chains, in the system, BI uses digital switches to activate the selected optimal RF chains. The digital switches are associated with the base band processing block, which is connected to a single RF chain. In this model, the number of data streams is the same as the number of RF chains. Subsequently, the number of data streams in the system is also optimized.

ii) Sub-problem Two: This problem is focused on maximizing the spectral efficiency of the system by designing the D-A precoder and combiner matrices with low complexity. The conventional way in [9], [10], [8], [6] is to avoid multi-variable joint optimization and decouple the transmitter and receiver, which adds complexity to the existing algorithms. In contrast, this paper introduces the concept of effective channel gains to realize the joint 
design of the transceiver, and investigate the design hybrid D-A system for low energy consumption. The analog beamformer is designed under non-convex constant modulus constraints based on two methods that exploit the potential of gaining the knowledge of the channel. The first method is called the eigenvalue decomposition (EVD), and is based on calculating the eigenvector that corresponds to the largest eigenvalue [11]. EVD achieves high spectral efficiency but is computationally complex because of the decomposition operation. The second method is called power iteration (PI) and based on calculating the eigenvector that corresponds to the largest eigenvalue, which avoids the decomposition operation. The main advantages of the EVD and PI methods are that both schemes: i) do not require calculating the gradient of the optimization function as utilized in existing algorithms [9], [8], [12], [6], which reduces the complexity of the system significantly; ii) do not require a high number of iterations, as they only calculate the eigenvector that corresponds to the largest eigenvalue; iii) avoid the use of the Euclidean distance optimization problem. Thereafter, the digital precoder is designed by proposing a low dimension matrix that is generated based on he duality of the uplink and downlink effective channel gains. The optimal digital precoder is obtained by applying SVD to a low dimensional effective uplink and downlink channel gain matrix.

b) Complexity Reduction: The proposed methods in this paper manage to reduce the computational complexity of the hybrid D-A system. The design of the analog part based on EVD only involves the number of antennas without the need for iterative optimization and SVD operations. Then, the PI algorithm is deployed to determine the highest eigenvalue and corresponding eigenvector without the need of a decomposition step. Besides, the digital part does not require a high dimension matrix as a low dimension matrix is proposing based on the effective uplink and downlink channel gains.

c) Phase Shifters Resolution: Since the power consumption increases with the resolution of the phase shifters of the analog precoder, a trade-off between the spectral efficiency and power consumption based on a limited resolution is investigated. This allows us to conclude the EE of the intended hybrid D-A system when utilizing different numbers of quantized bits.

- Does the abstract give a clear summary of the results of the paper? No. The novelty of this paper is not presented clearly in the abstract.

Response: Thanks for your suggestion, we have tried to improve the overall presentation of the abstract significantly by considering all the editor's and reviewers' comments.

Hybrid digital to analog (D-A) precoding is widely used in millimeter wave systems to reduce the 
power consumption and implementation complexity incurred by the number of radio frequency (RF) chains that consume a lot of the transmitted power in this system. In this paper, an optimal number of RF chains is proposed to achieve the desired energy efficiency (EE). Here, the optimization problem is formulated in terms of fractional programming maximization, resulting in a method with a twofold novelty: First, the optimal number of RF chains is determined by the proposed bisection algorithm, which results in optimized number of data streams. Second, the optimal analog precoders/combiners are designed by eigenvalue decomposition and power iteration algorithm, followed by the digital precoders/combiners which are designed based on the singular value decomposition to the proposed effective uplink and downlink channel gains. Furthermore, the proposed D-A systems are designed carefully to attain a lower complexity than the existing D-A algorithms while achieving reasonable performance. Finally, the impact of utilizing a different number of quantized bits of resolution on the EE is investigated. Simulation results show that the proposed algorithms outperform existing algorithms in terms of EE, spectral efficiency, and computational complexity. 


\section{Reviewer 2 Comments}

\section{a) The reviewer thinks the paper can be accepted. But the authors should carefully proofread the manuscript to remove all typos.}

Response: We appreciate your effort and thank you for your careful reading. We have proofread the paper and fixed numerous grammar and presentation issues.

The Authors

\section{REFERENCES}

[1] M. Alonzo, S. Buzzi, A. Zappone, and C. D’Elia, “Energy-Efficient Power Control in Cell-Free and User-Centric Massive MIMO at Millimeter Wave," IEEE Transactions on Green Communications and Networking, vol. 3, pp. 651-663, Sep. 2019.

[2] J. Opadere, Q. Liu, T. Han, and N. Ansari, "Energy-Efficient Virtual Radio Access Networks for Multi-Operators Cooperative Cellular Networks," IEEE Transactions on Green Communications and Networking, vol. 3, pp. 603-614, Sep. 2019.

[3] S. Buzzi, C. I, T. E. Klein, H. V. Poor, C. Yang, and A. Zappone, "A Survey of Energy-Efficient Techniques for 5G Networks and Challenges Ahead," IEEE Journal on Selected Areas in Communications, vol. 34, pp. 697-709, April 2016.

[4] S. Hur, S. Baek, B. Kim, Y. Chang, A. F. Molisch, T. S. Rappaport, K. Haneda, and J. Park, "Proposal on Millimeter-Wave Channel Modeling for 5G Cellular System," IEEE J. Sel. Areas in Signal Processing, vol. 10, pp. 454-469, April 2016.

[5] O. Alluhaibi, E. Kampert, P. A. Jennings, and M. D. Higgins, "Impact of Overlapped AoAs on the Achievable Uplink Rate of Hybrid Beamforming for Massive MIMO mm-Wave Systems for Industrial Environments,” IEEE Access, vol. 7, pp. 101178101194, 2019.

[6] A. Kaushik, J. Thompson, E. Vlachos, C. Tsinos, and S. Chatzinotas, "Dynamic RF Chain Selection for Energy Efficient and Low Complexity Hybrid Beamforming in Millimeter Wave MIMO Systems," IEEE Transactions on Green Communications and Networking, pp. 1-1, 2019.

[7] R. Zi, X. Ge, J. Thompson, C. Wang, H. Wang, and T. Han, "Energy Efficiency Optimization of 5G Radio Frequency Chain Systems," IEEE Journal on Selected Areas in Communications, vol. 34, pp. 758-771, April 2016.

[8] X. Yu, J. C. Shen, J. Zhang, and K. B. Letaief, "Alternating Minimization Algorithms for Hybrid Precoding in Millimeter Wave MIMO Systems," IEEE J. Sel. Topics in Signal Processing, vol. 10, pp. 485-500, Apr. 2016.

[9] O. El Ayach, S. Rajagopal, S. Abu-Surra, Z. Pi, and R. W. Heath Jr., "Spatially sparse precoding in millimeter wave MIMO systems,” IEEE Trans. on Wireless Commun., vol. 13, pp. 1499-1513, Mar. 2014.

[10] X. Gao, L. Dai, S. Han, C. L. I, and R. W. Heath, "Energy-Efficient Hybrid Analog and Digital Precoding for MmWave MIMO Systems With Large Antenna Arrays," IEEE Jour.l on Sel. Areas in Commui., vol. 34, pp. 998-1009, Apr. 2016.

[11] A. Goldsmith, Wireless Communications. New York, NY, USA: Cambridge University Press, 2005.

[12] O. E. Ayach, R. W. Heath, S. Abu-Surra, S. Rajagopal, and Z. Pi, "The capacity optimality of beam steering in large millimeter wave MIMO systems," in IEEE 13th International Workshop on Signal Processing Advances in Wireless Commun. (SPAWC), pp. 100-104, Jun. 2012. 